\title{
Linx
}

Revue des linguistes de l'université Paris X Nanterre

39 | 1998

Modèles linguistiques : convergences, divergences

\section{Verbes « à montée » et auxiliaires dans une grammaire d'arbres adjoints}

Raising verbs and auxiliaries in a Tree Adjoining grammar

\section{Anne Abeillé}

\section{OpenEdition}

1 Journals

Édition électronique

URL : http://journals.openedition.org/linx/882

DOI : 10.4000/linx.882

ISSN : 2118-9692

Éditeur

Presses universitaires de Paris Nanterre

\section{Édition imprimée}

Date de publication : 15 décembre 1998

Pagination : 119-158

ISSN : 0246-8743

\section{Référence électronique}

Anne Abeillé, « Verbes « à montée » et auxiliaires dans une grammaire d'arbres adjoints », Linx [En ligne], 39 | 1998, mis en ligne le 28 juin 2012, consulté le 10 décembre 2020. URL : http:// journals.openedition.org/linx/882 ; DOI : https://doi.org/10.4000/linx.882

Ce document a été généré automatiquement le 10 décembre 2020.

Département de Sciences du langage, Université Paris Ouest 


\section{Verbes « à montée » et auxiliaires dans une grammaire d'arbres adjoints $^{1}$}

Raising verbs and auxiliaries in a Tree Adjoining grammar

Anne Abeillé

1 Je voudrais reprendre ici la question rebattue des verbes dits «à montée » en français. Caractériser une classe de verbes par une transformation syntaxique ne va pas de soi et doit être logiquement remis en cause dans le cadre d'une théorie linguistique non transformationnelle. Dans la plupart des grammaires d'unification (LFG, GPSG ou HPSG), la distinction entre verbes "à montée " et verbes "à contrôle " est déplacée en sémantique: la distinction n'est pas entre deux types de complémentation mais entre deux types de prédicat: ceux qui assignent un rôle sémantique à chacun de leurs arguments syntaxiques et ceux qui n'en assignent pas. De fait, les propriétés les plus généralement retenues comme caractéristiques des verbes dits "à montée » sont des propriétés sémantiques (non assignation d'un rôle sémantique à un argument syntaxique, possibilité d'un argument idiomatique ou non référentiel). Dans le cadre d'une syntaxe "surfaciste ", on devrait donc logiquement abandonner la dénomination de verbe "à montée ", qui constitue un archaïsme terminologique, et lui préférer celle de verbe « transparent » (Langacker 1995) ou sémantiquement déficient ${ }^{2}$.

Mais une caractérisation purement sémantique pose elle-même plusieurs types de problèmes, empiriques et théoriques:

1) Est-il bien vrai que les verbes «transparents » n'ont aucune propriété syntaxique qui les distingue? C'est une question empirique beaucoup débattue (cf. en français Ruwet 1972, 1983, Rooryck 1990, Baschung 1998 etc) que je reprendrai ici en identifiant certaines déficiences syntaxiques de ces verbes,

2) Quelle analyse adopter pour les verbes auxiliaires ? Peut-on les ranger (comme en LFG, en GPSG ou en HPSG) dans la classe des verbes «à montée » ? Sémantiquement, ils sont à la fois « transparents » vis-à-vis de leur sujet et non prédicatifs. Syntaxiquement, il faut 
distinguer être et avoir, d'une part, qui ont des propriétés spécifiques et aller et venir d'autre part, qui se comportent comme les verbes « à montée " prédicatifs,

3) Comment un verbe "transparent» peut-il avoir en syntaxe un sujet (ou un complément) auquel il n'assigne pas de rôle sémantique? Dans une théorie transformationnelle, on peut maintenir en structure profonde un principe de correspondance entre arguments syntaxiques et arguments sémantiques, c'est-à-dire que tous les arguments syntaxiques référentiels doivent recevoir un rôle sémantique du prédicat qui les gouverne localement. Dans la plupart des grammaires d'unification, le lien entre arguments syntaxiques et arguments sémantiques est plus lâche.

Le débat ne se réduit donc pas à trancher entre analyse transformationnelle et analyse surfaciste; il s'agit autant de déterminer quels principes guident l'interface syntaxesémantique : peut-on maintenir que la structure argumentale (sémantique) détermine la sous-catégorisation syntaxique?

7 Je présenterai ici l'analyse des auxiliaires de temps et des verbes «à montée » adoptée en grammaire d'arbres adjoints (TAG). Cette analyse est originale en ce qu'elle maintient une distinction syntaxique entre montée et contrôle (et une contrainte de coïncidence entre argument syntaxique (référentiel) et argument sémantique (direct)) sans avoir besoin de recourir à des transformations syntaxiques. Elle ne résout évidemment pas tout mais permet de prédire certaines propriétés parfois négligées de ces verbes en français.

8 Je rappellerai d'abord (\$1) les principes et les difficultés de l'analyse transformationnelle, avant de reprendre la question des propriétés syntaxiques des verbes «à montée » en français (\$2). Je présenterai ensuite l'analyse adoptée en TAG (\$3), avant de la comparer aux analyses proposées en HPSG (\$4).

\section{L'analyse transformationnelle des verbes « à montée »}

\subsection{L'explication syntaxique d'une déficience sémantique}

Dès les débuts de la grammaire générative (Rosenbaum (1967), Perlmutter (1970)), une distinction entre deux types de verbes à complément infinitif a été proposée, pour l'anglais, avec d'un côté les verbes dits "à montée du sujet " (abrégé en "à montée ») tels que sembler, pouvoir, commencer à... (en français), de l'autre les verbes dits «à contrôle » tels que vouloir, promettre, persuader... A la base de la distinction, on trouve essentiellement une propriété sémantique : les verbes « à montée » n'assignent pas de rôle sémantique au sujet. Ce sont soit des marqueurs de temps ou d'aspect (aller, commencer...), soit des prédicats unaires qui prennent toute la proposition (sujet compris) comme argument sémantique (sembler), tandis que les verbes «à contrôle » ont au moins deux arguments sémantiques (le sujet et l'infinitive). On a donc synonymie en (1) mais pas en (2):

\begin{tabular}{|l|l|l|l|}
\hline & (1) & a. & Jean semble / peut apprécier les enfants \\
\hline & $=$ & b. & Les enfants semblent / peuvent être appréciés par Jean \\
\hline & (2) & a. & Jean veut / essaie d'apprécier les enfants \\
\hline
\end{tabular}


La différence de sujet entre les phrases (a) et (b) ne change pas l'interprétation avec un verbe «à montée » (en (1)) : il suffit que les infinitives soient synonymes. Avec un verbe « à contrôle » au contraire, comme en (2), l'interprétation n'est plus la même, à partir du moment où le sujet syntaxique, et donc sémantique, de vouloir ou essayer n'est pas le même.

11 Conséquence : les verbes « à montée » sont transparents pour la sélection du sujet (et sont donc compatibles avec un sujet idiomatique ou impersonnel, cf. (3)), alors que les verbes « à contrôle » imposent des restrictions (et prennent en général un sujet agentif, cf. (4)) ${ }^{3}$ :

\begin{tabular}{|l|l|l|l|}
\hline & (3) & a. & Paul semble / peut / commence à dormir \\
\hline & & b. & Il semble / peut / commence à pleuvoir \\
\hline & & c. & La moutarde semble / peut / commence à monter au nez de Paul \\
\hline & (4) & a. & Paul veut / essaie de dormir \\
\hline & & b. & *Il veut / essaie de pleuvoir \\
\hline & & c. & $*$ La moutarde veut / essaie monter au nez de Paul \\
\hline
\end{tabular}

12 La sélection du sujet des verbes « à montée » est entièrement faite par l'infinitif qui suit, alors qu'elle est faite conjointement par les deux verbes dans le cas des verbes «à contrôle ».

13 Autre conséquence : il y a une relation de paraphrase entre constructions à montée et tours impersonnels à complément phrastique :

\begin{tabular}{|l|l|l|l|}
\hline & (5) & a. & Paul semble dormir \\
\hline & & b. & Il semble que Paul dort \\
\hline
\end{tabular}

14 De façon en apparence paradoxale, la grammaire générative donne une explication purement syntaxique de ces propriétés sémantiques. Sans entrer dans le détail de toutes les versions proposées, l'analyse transformationnelle est la suivante. Les verbes «à montée » et les verbes «à contrôle » ont des structures profondes différentes : les verbes «à contrôle » ont à la fois un sujet et un complément phrastique (les infinitives étant toujours analysées comme des phrases), tandis que les verbes «à montée » ont seulement un complément phrastique (et pas de sujet). Dans le modèle du gouvernement et du liage (GB), la différence entre structures profondes (6) est conservée au niveau des structures de surface (7). Les phrases «à montée » sont dérivées par une règle de mouvement (qui déplace le sujet enchâssé en position de sujet principal ), alors que les phrases à contrôle mettent simplement en jeu une opération de coöndiciation entre le sujet vide (PRO) de 
l'infinitif et le sujet de veut (ou d'un autre argument) selon les principes de liage (Chomsky 1981) :

\begin{tabular}{|l|l|l|l|}
\hline & (6) & a. & {$\left[_{N P}\right.$ e] semble $\left[{ }_{S}\right.$ Jean partir $]$} \\
\hline & & b. & Jean veut $\left[\left[_{S}\left[_{S} P R O\right.\right.\right.$ partir $\left.]\right]$ \\
\hline & (7) & a. & {$\left[{ }_{N P}\right.$ Jean $\left._{i}\right]$ semble $\left[{ }_{S}\right.$ e partir $]$} \\
\hline & & b. & Jean $_{i}$ veut $\left[{ }_{S}\left[{ }_{S} P R O_{i}\right.\right.$ partir $\left.]\right]$ \\
\hline
\end{tabular}

La transformation qui déplace le sujet de (6)a à (7)a est obligatoire et ne résulte pas d'une règle spécifique : en posant que tout SN lexicalement réalisé doit recevoir un cas et qu'un infinitif ne peut assigner le cas nominatif, on explique que Jean doive « monter » en (6)a pour recevoir (de semble) le cas nominatif. D'autres différences apparaissent entre (7)a et (7)b : la catégorie vide (trace) laissée par le déplacement en (7)a n'est pas de même nature que le pronom nul de (7)b; conséquence : la frontière syntagmatique est plus « fine » (S) après semble qu'après veut $\left(\mathrm{S}^{\prime}\right)$ car semble doit pouvoir « gouverner » cette catégorie vide.

\subsection{Les difficultés de l'analyse transformationnelle}

16 L'analyse transformationnelle des verbes "à montée » rencontre des difficultés bien connues ${ }^{4}$. L'hypothèse d'une complémentation phrastique correspondant à la structure profonde est fragile. Cette complémentation est attestée sur quelques verbes seulement qui font figure de curiosité dans l'ensemble des verbes concernés, comme cela a été noté pour le français par Gross (1975) (et pour l'anglais par Bresnan (1982)).

\begin{tabular}{|l|l|l|l|}
\hline & (8) & a. & Jean finit de travailler \\
\hline & & b. & * Il finit que Jean travaille \\
\hline & (9) & a. & Jean va travailler \\
\hline & & b. & *Il va que Jean travaille \\
\hline
\end{tabular}

En français, seuls sembler, paraître, s'avérer, se trouver et les passifs « transparents » (être dit, être supposé....) entrent dans la structure il $V$ que $P$. Cette absence de complémentation phrastique s'explique assez bien à partir du moment où les verbes concernés sont très généralement non prédicatifs. Sémantiquement, ils sont plutôt du côté des auxiliaires (modaux : pouvoir, devoir.., aspectuels : commencer, finir... ou temporels : aller, venir ...) et c'est une donnée que l'analyse transformationnelle ignore totalement.

Une autre difficulté est l'assignation casuelle par le verbe "à montée »: si le sujet de l'infinitif doit «monter » pour recevoir le cas nominatif, on explique mal les données dans des langues où il existe des sujets à d'autres cas (cf. sujets datifs ou génitifs en islandais): dans ces langues, le cas observé pour le sujet dans les constructions «à 
montée » n'est pas forcément le nominatif mais peut être celui assigné par l'infinitif (cf. Andrews 1982, Sag et al. 1992).

Pour le français, le placement sur l'infinitif du clitique en complément du sujet a été parfois considéré avec Ruwet (1972) comme le «meilleur» argument pour la transformation de montée, mais il ne va pas non plus sans difficultés (cf. Tasmowski 1990). Ruwet (1972) interprète le contraste entre (10)b et (10)c comme témoignant de l'appartenance « profonde » du sujet à l'infinitive :

\begin{tabular}{|l|l|l|l|}
\hline & $(10)$ & a. & La fin de ce livre semble / doit être géniale \\
\hline & & b. & La fin semble / doit en être géniale \\
\hline & & c. & ? ? La fin en semble / doit être géniale \\
\hline
\end{tabular}

L'analyse de Ruwet repose crucialement sur l'application de la pronominalisation du complément du sujet avant la montée de celui-ci ${ }^{5}$ :

\begin{tabular}{|l||l|l|}
\hline & $\varnothing$ semble ( (la fin-SP) être géniale) \\
\hline cliticisation $->$ & $\varnothing$ semble ((la fin- $\left.\mathrm{e}_{\mathrm{i}}\right)$ en $\mathrm{n}_{\mathrm{i}}$ être géniale $)$ \\
\hline montée $->$ & $\left(\text { la fin- } \mathrm{e}_{\mathrm{i}}\right)_{\mathrm{j}}$ semble $\left(\mathrm{e}_{\mathrm{j}}\right.$ en $\mathrm{n}_{\mathrm{i}}$ être géniale $)$ \\
\hline
\end{tabular}

21 Mais on comprend mal pourquoi toute pronominalisation est exclue avec un verbe «à contrôle » :

\begin{tabular}{|l|l|l|l|}
\hline & (11) & a. & L'auteur de ce livre prétend / dit être génial \\
\hline & & b. & *L'auteur prétend / dit en être génial \\
\hline & & c. & ?? L'auteur en prétend / dit être génial \\
\hline
\end{tabular}

22 Comme le montre Tasmowski (1990), les contraintes sur la cliticisation d'un complément du sujet sont plutôt d'ordre pragmatique (le complément doit être plus 'topique' que le sujet) et c'est le caractère humain (ou agentif) du sujet des verbes à contrôle qui empêche cette cliticisation ${ }^{6}$. Comme le montre lui-même Ruwet $(1983,1991)$, certains verbes à contrôle, pris au sens figuré avec un sujet non agentif, autorisent le placement sur l'infinitif d'un en lié au sujet :

\begin{tabular}{|l|l|l|l|l|}
\hline & $(12)$ & a. & La liste de ces verbes ne prétend pas être exhaustive \\
\hline & & b. & La liste ne prétend pas en être exhaustive \\
\hline (13) & a. & La deuxième partie de Sein und Zeit attend encore d'être publiée \\
\hline
\end{tabular}


Les données de (10) ne fournissent donc pas un argument en faveur de la structure syntaxique profonde (6a).

Un autre problème, pour le français, mentionné par Kayne (1980) et Rooryck (1990), concerne l'analyse de à et de après verbe à montée : à partir du moment où l'infinitive est analysée comme $\mathrm{S}$ (et non $\mathrm{S}^{\prime}$ ), à et de ne peuvent être des complémenteurs. Mais, il est difficile d'en faire des prépositions, puisque cela empêcherait aussi le verbe à montée de gouverner la trace du sujet de l'infinitif et que, d'autre part, l'infinitive n'a pas les propriétés attendues d'un complément prépositionnel :

\begin{tabular}{|l|l|l|l|}
\hline & (14) & a. & Il vient de pleuvoir \\
\hline & & $b$. & *Il en vient \\
\hline & (15) & a. & Paul commence à comprendre \\
\hline \hline & & b. & *Paul y commence \\
\hline
\end{tabular}

Kayne (1980) propose donc de ne retenir comme verbes à montée que ceux qui se construisent directement avec l'infinitif, créant ainsi un contraste syntaxique artificiel entre aller et venir de par exemple.

Dernier problème, celui de l'analyse des auxiliaires. La grammaire générative les a d'abord mis sous un nœud AUX (ou Infl) spécifique, suivant en cela la grammaire traditionnelle qui les étudie au chapitre de la morphologie verbale. A partir de Chomsky (1986), ils sont analysés comme des têtes fonctionnelles, sans contenu prédicatif, qui prennent un complément SV sans lui assigner de rôle sémantique. Ceci explique bien qu'ils ne puissent entrer dans une structure à complément phrastique, mais l'adoption des têtes fonctionnelles, qui s'étend aux affixes de temps, conduit à des règles syntaxiques ad hoc de placement d'affixes et à une remise en cause contestable du partage entre morphologie et syntaxe. De plus, la même analyse devrait assimiler en français les auxiliaires de temps être et avoir (suivis d'un participe) et les temporels aller et venir (suivis d'un infinitif): comment dans ces conditions expliquer leurs propriétés syntaxiques différentes?

On peut se demander pourquoi une transformation si contestable jouit d'une telle longévité (elle figure toujours en bonne place dans les manuels de syntaxe). L'intérêt de l'analyse transformationnelle est de maintenir (en structure profonde) un parallèlisme strict entre arguments sémantiques et arguments syntaxiques (référentiels). Cette motivation profonde est illustrée par le critère thématique ( $\theta$-critère) qu'on peut présenter ainsi :

- un prédicat assigne un rôle thématique à chacun de ses arguments syntaxiques (dont la position est occupée par une expression référentielle),

- chaque expression référentielle reçoit un rôle thématique du prédicat dont elle est un argument syntaxique. 
C'est l'incapacité du sujet du verbe «à montée » à recevoir de celui-ci un rôle thématique qui empêche d'y voir un sujet syntaxique profond. La spécificité de la structure syntaxique associée aux verbes à montée n'est donc pas seulement le reflet d'une vision " hégémonique » de la syntaxe (leur spécificité sémantique doit avoir une explication syntaxique), elle est la conclusion naturelle de cette contrainte d'interface. C'est dire qu'elle peut sembler la seule solution pour tout linguiste qui pense que la structure argumentale (sémantique) est un bon guide pour déterminer la sous-catégorisation (syntaxique) des items lexicaux ${ }^{7}$. Sans trancher ce débat qui dépasse le cadre de cet article, il faut reconnaître que c'est là un argument en faveur de l'analyse transformationnelle.

Avant de proposer une analyse surfaciste qui préserve cette contrainte d'interface, revenons d'abord sur quelques propriétés syntaxiques des auxiliaires et des verbes «à montée » en français.

\section{Quelques propriétés syntaxiques des verbes « à montée »}

La question des propriétés syntaxiques des verbes «à montée » a été maintes fois abordée en français, avec parfois des affirmations contradictoires (cf. notamment Ruwet 1972, 1983, Huot 1974, Gross 1975, Rochette 1988, Rooryck 1989, 1990, Tasmowski 1980, 1990). Parmi les études les plus récentes, on peut citer Baschung (1998) qui contraste systématiquement constructions à montée et à contrôle: pour elle, les propriétés syntaxiques sont éparses si l'on regarde plus de deux verbes, et toute tentative de caractérisation syntaxique est infructueuse; il faudrait donc se rabattre sur des tests purement sémantiques et on aboutit à un continuum entre "montée " et "contrôle " (selon que les verbes ont un argument contrôleur plus ou moins agentif) $)^{8}$.

31 Il est vrai que la classe des verbes «à montée » comme celle des verbes «à contrôle » ne sont pas homogènes, et que les données sont difficiles : d'une part, les verbes en jeu sont souvent polysémiques, d'autre part, ils entrent souvent dans plusieurs constructions concurrentes. Je me limiterai ici (faute de place) aux verbes «à montée du sujet en position sujet " (abrégés en "à montée ») suivis d'un infinitif ${ }^{9}$ et je ne choisirai que quelques propriétés syntaxiques pour les comparer aussi bien aux verbes « à contrôleur sujet » qu'aux auxiliaires de temps avoir et être ${ }^{10}$. Il me semble qu'on peut dégager d'une part des régularités, d'autre part des tendances suffisamment fortes pour être significatives.

\subsection{Les propriétés communes avec les verbes à contrôle}

On observe, pour les verbes "à montée", (au moins) deux propriétés syntaxiques communes avec les verbes "à contrôle" et différentes des auxiliaires de temps: le placement des clitiques compléments et celui de la négation.

\subsubsection{Le placement des clitiques compléments}

33 Comme dans les autres langues romanes, les pronoms clitiques se placent en français sur l'auxiliaire et non sur le participe : 
(16) Jean l'a vu /* a le vu

En français moderne, ils se placent sur l'infinitif après les verbes à contrôle comme après les verbes à montée (tandis que dans d'autres langues romanes, ils peuvent se placer aussi sur les verbes à montée et sur quelques verbes à contrôle, appelés verbes « restructurants ») :

\begin{tabular}{|l|l|l|l|l|}
\hline & (17) & a. & Jean veut le voir $/{ }^{*}$ le veut voir \\
\hline & & b. & Jean promet de le finir $/{ }^{*}$ le promet de finir \\
\hline & (18) & a. & Jean peut le voir $/ *$ le peut voir \\
\hline & & b. & Jean vient de le finir $/ *$ le vient de finir \\
\hline
\end{tabular}

\subsubsection{La négation de l'infinitive}

Il est bien connu que la négation se place sur l'auxiliaire et non sur le participe. Pour les verbes à contrôle, les deux positions sont possibles avec un sens différent :

\begin{tabular}{|l|l|l|l|}
\hline & $(19)$ & a. & Jean n'a pas pleuré \\
\hline & & b. & *Jean a ne pas pleuré \\
\hline & $(20)$ & a. & Jean n'aimerait pas aller au cirque \\
\hline & & b. & Jean aimerait ne pas aller au cirque \\
\hline
\end{tabular}

Contrairement à ce qu'affirmait notamment Gross (1975), il est également possible de nier l'infinitif après un verbe "à montée " même si cette négation est moins naturelle que celle du verbe « à montée $»^{11}$ :

\begin{tabular}{|l|l|l|l|l|}
\hline & $(21)$ & a. & Jean semble ne pas avoir compris \\
\hline & & $b$ & Jean ne semble pas avoir compris \\
\hline & & & & Tu va voir, ça va encore ne pas marcher! \\
\hline & & b. & Ça ne va encore pas marcher! \\
\hline & & a. & Ils recommencent à ne pas répondre au téléphone \\
\hline & & b. & Ils ne recommencent pas à répondre au téléphone \\
\hline
\end{tabular}


Comme avec les verbes à contrôle, la négation de l'infinitif a souvent un sens différent de celle du verbe principal (cf. 23) et il est possible de cumuler ces deux négations (cf. 24) :

\begin{tabular}{|l|l|l|l|}
\hline & (24) & a. & Jean ne risque pas de ne pas réussir. \\
\hline & & b. & Jean ne peut pas ne pas réussir. \\
\hline
\end{tabular}

En dépit de ces différences, les verbes «à montée » partagent avec les auxiliaires de temps certaines propriétés qui mettent en doute leur assimilation syntaxique avec les verbes à contrôle.

\subsection{Les propriétés communes avec les auxiliaires de temps}

39 Si l'on admet que l'existence d'idiosyncrasies et de variations entre locuteurs n'est pas un obstacle à la formulation de généralisations syntaxiques, on constate que les verbes «à montée » (à la différence des verbes "à contrôle») font massivement preuve d'une déficience syntaxique analogue à celle des auxiliaires de temps (et des verbes restructurants dans les autres langues romanes). En effet, ils n'acceptent pas de complément indépendamment de leur complément verbal, et celui-ci n'a pas toutes les propriétés syntaxiques attendues d'un complément ${ }^{12}$.

\subsubsection{Pas d'autre complément que l'infinitive}

Les auxiliaires de temps ne peuvent pas avoir de complément indépendant du participe passé ; les verbes à contrôle peuvent avoir un complément indépendant de l'infinitif :

\begin{tabular}{|l|l|l|l|}
\hline & $(25)$ & a. & Paul promet (à Marie) de venir \\
\hline & & b. & Paul apprend (de Marie) à jouer du piano \\
\hline
\end{tabular}

41 L'absence de complément indépendant est frappante pour les verbes « à montée » ayant un autre emploi non transparent. Ruwet (1972) distingue ainsi deux verbes promettre et deux verbes menacer, l'un à sujet agentif (à contrôle, a), l'autre transparent (à montée, b) ${ }^{13}$ :

\begin{tabular}{|l|l|l|l|}
\hline & (26) & a. & Jean a promis de venir demain \\
\hline & & b. & Ça promet de barder \\
\hline & (27) & a. & Les terroristes ont menacé de tout faire sauter \\
\hline & & b. & Le lait menace de déborder \\
\hline
\end{tabular}


Seul l'emploi à sujet agentif dénote une promesse ou une menace, l'emploi transparent dénote simplement la probabilité de l'évènement. Comme le note Ruwet (1972), l'emploi transparent n'est pas compatible avec un second complément nominal ou prépositionnel :

\begin{tabular}{|l|l|l|l|}
\hline & $(28)$ & a. & Jean a promis à tous de venir demain \\
\hline & & b. & * Ça promet à tous de barder \\
\hline & $(29)$ & a. & Les terroristes ont menacé la famille de tout faire sauter \\
\hline & & b. & * Le lait menace la famille de déborder \\
\hline
\end{tabular}

Comme l'auxiliaire de temps, le verbe "à montée » n'admet pas d'autre complément que le verbe qui suit et les compléments de celui-ci. Seuls sont possibles, indépendamment de l'infinitif, des clitiques figés incorporés au verbe à montée : se mettre à, s'avérer, se trouver ${ }^{14}$.

\subsubsection{L'infinitive n'a pas toutes les propriétés d'un complément}

En reprenant les propriétés les plus typiques, c'est-à-dire le clivage et la pronominalisation, il est clair que la séquence verbale qui suit l'auxiliaire de temps n'a pas les propriétés attendues d'un complément. Après les verbes "à montée " (et à la différence des verbes « à contrôle »), l'infinitive échoue généralement aux mêmes tests.

\subsubsection{Clivage de l'infinitive}

La pseudo-clivée ${ }^{15}$ de la séquence participiale est impossible après un auxiliaire $(30, \mathrm{cf}$. Abeillé \& Godard 1996), celle de l'infinitive est en général bien acceptée avec les verbes « à contrôle » (cf. 31).

\begin{tabular}{|l|l|l|l|}
\hline & $(30)$ & $a$. & *Ce que Paul a, c'est bu du vin \\
\hline & & b. & *Ce que Paul est, c'est parti hier \\
\hline (31) & a. & Ce que Jean a promis, c'est d'aller chez Marie \\
\hline & & b. & Ce que Jean aime, c'est (d') aller au cinéma \\
\hline
\end{tabular}

Elle est généralement impossible avec les verbes « à montée » (32-34) :

\begin{tabular}{|l|l|l|l|}
\hline & $(32)$ & a. & Jean semble travailler nuit et jour. \\
\hline & & b. & * Ce que Jean semble, c'est travailler nuit et jour \\
\hline & (33) & a. & Le lait menace de déborder \\
\hline & & b. & * Ce que / dont le lait menace, c'est de déborder \\
\hline
\end{tabular}




\begin{tabular}{|l|l|l|l|}
\hline & (34) & a. & Jean est en train de rédiger sa thèse \\
\hline & & b. & * Ce que Jean est en train, c'est de rédiger sa thèse \\
\hline & (35) & a. & La pluie commence à tomber \\
\hline & & b. & * Ce que/à quoi la pluie commence, c'est à tomber \\
\hline
\end{tabular}

On attend la reprise du proverbe faire (avec un infinitif agentif) :

\begin{tabular}{|l|l|l|l|}
\hline & (36) & a. & Ce que Jean semble faire, c'est travailler nuit et jour \\
\hline & & b. & Ce que Jean est en train de faire, c'est de rédiger sa thèse \\
\hline
\end{tabular}

Baschung (1998) note deux exceptions à cette généralisation, pouvoir et risquer, avec les exemples suivants :

\begin{tabular}{|l|l|l|l|}
\hline & (37) & a. & Ce que peut Jean, c'est travailler jour et nuit \\
\hline & & b. & Ce que risque Jean, c'est de devoir travailler jour et nuit \\
\hline
\end{tabular}

Mais si l'on prend un sujet non agentif, ou non référentiel, le tour devient inacceptable :

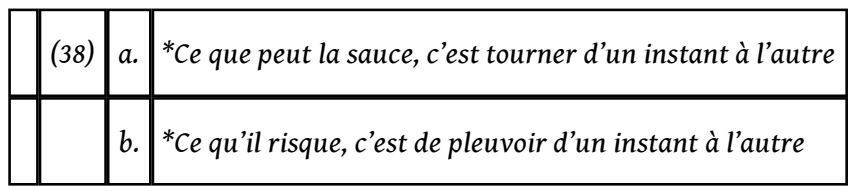

On peut donc admettre qu'on a affaire en (37) à un emploi spécifique de pouvoir ou risquer qui n'est pas celui du verbe transparent (ou à montée). L'ambiguïté du verbe pouvoir a été maintes fois notée (cf. Sueur 1979, Kayne 1977). Grossièrement, on note une bonne corrélation entre l'interprétation épistemique (être probable) et l'emploi transparent (ou à montée) d'une part, entre l'interprétation déontique ( = avoir la capacité, la permission, la possibilité de) et un emploi à sujet agentif d'autre part, même s'il peut y avoir certaines interférences. De la même façon, on peut distinguer le sens épistémique (probabilité) de risquer de son sens actif ( =prendre un risque ${ }^{16}$. Si l'on réserve l'analyse « à montée » à la première interprétation, les exemples (37) ne sont donc pas des contre-exemples.

\subsubsection{Pronominalisation de l'infinitive}

51 Le remplacement de l'infinitive par un pronom fort (cela) est toujours possible avec un verbe «à contrôle » (39) et impossible après un auxiliaire de temps (40) ou un verbe « à montée » (41, cf. Kayne 1977, Rochette 1988) : 


\begin{tabular}{|l|l|l|l|}
\hline & $(39)$ & a. & Jean ne veut plus aller voir Marie mais il avait promis cela \\
\hline & & b. & Aller au cinéma, Jean aime cela \\
\hline$(40)$ & a. & *Jean a mangé des fraises mais Marie n'a pas cela \\
\hline & & b. & * Allé au cinéma, Jean est cela. \\
\hline$(41)$ & a. & * Marie ne peut pas travailler jour et nuit mais elle devrait cela \\
\hline & & b. & *Marie ne veut pas travailler jour et nuit mais elle pourrait cela \\
\hline$(42)$ & a. & *Jean va aller à Rome mais Marie ne va pas cela \\
\hline & & b. & *Les rosiers semblent avoir soif mais les azalées ne semblent pas cela \\
\hline & c. & *Le lait va déborder mais la soupe ne menace pas (de) cela \\
\hline
\end{tabular}

On pourrait penser que c'est l'impossibilité d'une complémentation nominale alternative qui bloque la pronominalisation. Mais si l'on considère les verbes aspectuels, le contraste est net entre la (bonne) pronominalisation de leur complément nominal et la (mauvaise) pronominalisation de leur infinitive ${ }^{17}$ :

\begin{tabular}{|l|l|l|ll|}
\hline & (43) & a. & Marie a fini le nettoyage d'automne mais sa sœur commencera cela demain \\
\hline & & b. & *Marie a fini d'avoir peur la nuit mais sa sœur commence cela \\
\hline & $(44)$ & a. & Marie a cessé le travail au noir mais sa sœur va continuer cela pendant tout l'été \\
\hline & & b. & *Marie a cessé d'avoir peur la nuit mais Laurent va continuer cela pendant tout l'été \\
\hline
\end{tabular}

53 Il y a donc là une déficience syntaxique propre à l'infinitive.

54 La pronominalisation clitique (le) a été plus discutée : elle est toujours impossible avec les auxiliaires de temps (cf. Abeillé \& Godard 1996), et généralement possible avec les verbes à contrôle (avec certaines exceptions cf. 46b) :

\begin{tabular}{|l|l|l|l|}
\hline & $(45)$ & a. & * Paul a bu du vin, mais Marie ne l'a pas \\
\hline & & b. & * Paul est parti hier mais Marie ne l'est pas \\
\hline (46) & a. & Jean ne veut plus aller voir Marie mais il l'avait promis \\
\hline & & b. & *Aller au cinéma, Jean l'aime \\
\hline
\end{tabular}

55 Elle est généralement mauvaise avec les verbes «à montée » (cf. Kayne 1977, Zribi-Hertz 1986, Rochette 1988, Blanche-Benveniste et al. 1991), sauf avec devoir et pouvoir : 


\begin{tabular}{|l|l|l|l|}
\hline & $(47)$ & a. & *Les feuilles du marronnier ont fini de tomber mais celles du chêne ne l'ont pas fini \\
\hline & & b. & *Marie a fini d'écrire sa thèse mais sa scur le commence à peine \\
\hline$(48)$ & & *Paul va partir à Rome mais Marie ne le va pas \\
\hline$(49)$ & a. & ? ? Les rosiers semblent avoir soif mais les azalées ne le semblent pas ${ }^{18}$ \\
\hline & b. & *Le lait risque de déborder mais la soupe ne le risque pas \\
\hline$(50)$ & a. & Paul a pu partir, mais Marie ne l'a pas pu \\
\hline & b. & Donner tout à l'école, qui le devrait? \\
\hline
\end{tabular}

56 Comme pour pouvoir, discuté supra, de nombreux travaux sur devoir (cf. Sueur (1979) et Huot (1974), Tasmowski (1980))ont montré qu'on devait distinguer entre une interprétation «épistémique » (éventualité, probabilité) et une interprétation « déontique » (avoir le devoir de). Sans revenir sur tous les tests discutés, on peut retenir que c'est seulement dans l'interprétation épistémique que devoir et pouvoir se comportent comme des verbes à «montée ». Grossièrement, on peut assimiler interprétation déontique et sujet agentif, seule l'interprétation épistémique autorisant un sujet non référentiel ${ }^{19}$ :

\begin{tabular}{|l|l|l|l|}
\hline & (51) & a. & Jean peut venir (ambigu) \\
\hline & & b. & Il peut pleuvoir demain (non ambigu) \\
\hline
\end{tabular}

57 On peut aisément vérifier que l'interprétation épistémique rend la cliticisation de l'infinitive impossible :

(52) * Pleuvoir demain, il le peut / doit.

58 A partir du moment où on distingue (au moins) deux verbes pouvoir et deux verbes devoir, dont seul celui à interprétation épistémique est transparent, il n'y a pas de contreexemple.

\subsubsection{Deux propriétés plus marginales}

Deux autres propriétés syntaxiques sont observées avec certains verbes « à montée ", qui les rapprochent des auxiliaires de temps (et des verbes restructurants des autres langues romanes). Il s'agit des tours à Infinitif et de la « montée » du passif. 


\subsubsection{Les tours à Inf}

En italien ou en français, à la différence de l'anglais (cf. Kayne 1977, Rizzi 1978), l'objet omis dans les tours en à Infinitif (après certains adjectifs comme difficile ou dans des relatives) doit être celui du premier infinitif (53a,b-54a) et ne doit pas être enchâssé $(54 b, c-53 c, d)$ :

\begin{tabular}{|l|l|l|l|}
\hline & (53) & a. & Je cherche une carte à envoyer aux enfants \\
\hline & & b. & Je cherche quelqu'un à envoyer parler aux enfants \\
\hline & & c. & * Je cherche une carte à dire à Jean d'envoyer. \\
\hline & & d. & * On m'a donné un travail à prévoir de finir pour demain. \\
\hline (54) & a. & Un gouvernement est difficile à organiser dès la sortie des urnes. \\
\hline & & b. & $?^{*}$ Ce problème est difficile à vouloir résoudre pour demain \\
\hline & c. & * Un gouvernement est difficile à promettre d'organiser dès la sortie des urnes \\
\hline
\end{tabular}

61 Ces tours sont acceptables avec un temps composé (55, cf. Abeillé \& Godard 1996) mais aussi, pour certains locuteurs, avec certains verbes «à montée » (56, cf. Kayne 1974-75 p. $69)$.

\begin{tabular}{|l|l|l|l|l|}
\hline & (55) & a. & On m’a donné un travail à avoir fini pour demain. \\
\hline & & b. & ? Un gouvernement est difficile à avoir (déjà) organisé dès la sortie des urnes. \\
\hline (56) & a. & ? Je cherche une carte à pouvoir envoyer aux enfants. \\
\hline & b. & ? (Pour moi) ce livre serait impossible à commencer à lire aujourd'hui. \\
\hline
\end{tabular}

En (55) l'objet omis est celui du participe, en (56) c'est celui d'envoyer, ou lire, pas celui de pouvoir ou commencer. Si l'on maintient la généralisation ci-dessus (l'objet omis est celui du premier infinitif) tout se passe comme si l'auxiliaire (et le verbe à montée) « héritait » du complément du participe (ou de l'infinitif) qui suit.

\subsubsection{La « montée » du passif}

63 Des tours passifs particuliers (cf. Grevisse 1988, \$742) se rencontrent avec certains verbes à montée et témoignent aussi d'un partage de complément entre le verbe à montée et l'infinitif. On peut appeler cela « montée du passif $»^{20}$ :

$$
\text { (57) } \text { a. La maison a fini d'être construite le mois dernier. }
$$




\begin{tabular}{|l|l|l|l|}
\hline & & b. & La maison a été finie de construire le mois dernier. \\
\hline & $(58)$ & & Le livre a été achevé d'imprimer le mois dernier. \\
\hline
\end{tabular}

En (57)b et (58), les aspectuels achever de, finir de apparaissent au passif alors que c'est l'infinitif qui est passivé si l'on suit la sous-catégorisation. Il y a déplacement de la morphologie passive sur le verbe à montée ${ }^{21}$. Tout se passe donc comme si finir (ou achever ) pouvait « hériter » du complément direct de construire (ou d'imprimer).

En conclusion, les verbes «à montée » français n'ont ni les mêmes propriétés syntaxiques que les auxiliaires être et avoir, ni les mêmes que les verbes "à contrôle ». Ils partagent avec les verbes à contrôle le placement des clitiques compléments sur l'infinitif et la possibilité de nier l'infinitif indépendamment du verbe principal. Ils partagent avec les auxiliaires de temps le fait de ne pas admettre de complément indépendant de leur complément verbal, et l'échec de celui-ci aux propriétés habituelles des compléments (clivage, pronominalisation) ${ }^{22}$. Comment rendre compte de ces propriétés dans une théorie non transformationnelle?

\subsection{Propriétés syntaxiques et structures de constituants}

Les théories non transformationnelles s'appuient sur les structures de constituants enrichies par des traits pour expliquer les propriétés syntaxiques. Dans le cas des verbes "à montée ", trois structures sont a priori envisageables (figure 1). Compte tenu de l'étude qui précède, on doit reconnaître qu'on a affaire à deux blocs de propriétés contradictoires ${ }^{23}$. Le premier bloc de propriétés (placement des clitiques et de la négation) - appelons-les propriétés I - montre que l'infinitive forme un domaine syntaxique à part entière, et c'est la structure à enchâssement syntagmatique (A) qui en rend compte (à partir du moment où l'on empêche les pronoms clitiques de sortir de leur domaine syntaxique). Le second bloc de propriétés (complémentation déficiente) appelons-les propriétés II - s'explique lui immédiatement si l'on adopte au contraire une structure telle que B ou C dans laquelle l'infinitive ne forme pas un constituant :

Figure 1. Les trois structures de constituants en concurrence

(A)

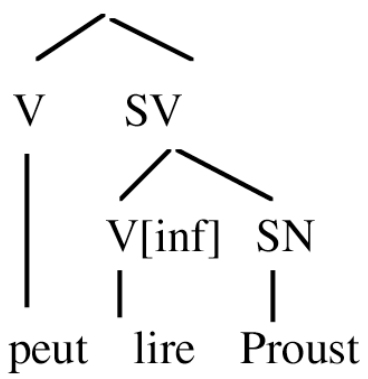

(B)

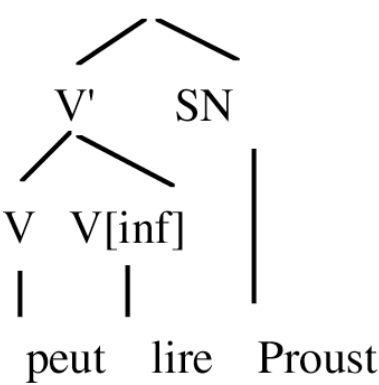

(C)

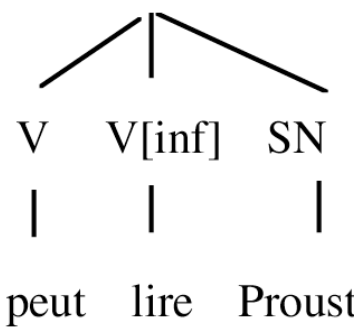

Admettons qu'une «bonne » théorie syntaxique doit rendre compte à la fois des deux blocs de propriétés considérés. Admettons également qu'elle ne doit assigner aux verbes en cause (une fois éliminés les cas d'ambiguïtés) qu'une seule structure syntaxique : dans ces conditions, laquelle choisir ${ }^{24}$ ? 

avant de revenir sur les analyses proposées en HPSG.

\section{L'analyse proposée en TAG}

71 Je ne présente ici que très brièvement les grammaires d'arbres adjoints (TAG) ${ }^{25}$ avant d'exposer l'analyse par adjonction des auxiliaires de temps et des verbes à montée en français.

\subsection{Les grammaires d'arbres adjoints lexicalisées} élémentaires associés aux mots dans le lexique et combinés par adjonction ou par substitution (selon qu'il s'agit d'un arbre auxiliaire ou initial). Les arbres élémentaires, qui définissent des unités syntaxiques et sémantiques, obéissent à des principes linguistiques généraux (Kroch \& Joshi 1985, Abeillé 1991, Frank 1992)26 :

- lexicalisation : tout arbre élémentaire a une feuille occupée par sa tête lexicale,

- les arbres élémentaires pour les constructions syntaxiquement apparentées (disponibles pour une même tête lexicale) sont regroupés en familles (ou reliés par des règles lexicales) : relatives, interrogatives, passif, impersonnel etc.

- consistance sémantique : les mots sans autonomie sémantique, tels que les complémenteurs, les prépositions régies ou les éléments figés des expressions idiomatiques, n'ancrent pas d'arbres élémentaires, ils sont co-têtes de l'arbre du prédicat qui les gouverne,

- coocurrence prédicat-argument : les arbres élémentaires dont est tête un mot prédicatif ont un nœud (feuille) pour chacun de ses arguments réalisés.

Pour le français, les arbres initiaux sont utilisés pour les noms, les verbes à arguments nominaux ou prépositionnels. Les arbres auxiliaires sont utilisés pour les modifieurs 
(relatives, adjectifs épithètes, adverbes, circonstants), les déterminants et les verbes à complétives. Des exemples d'arbres élémentaires sont les suivants ${ }^{27}$ :

Figure 2. Exemples d'arbres élémentaires

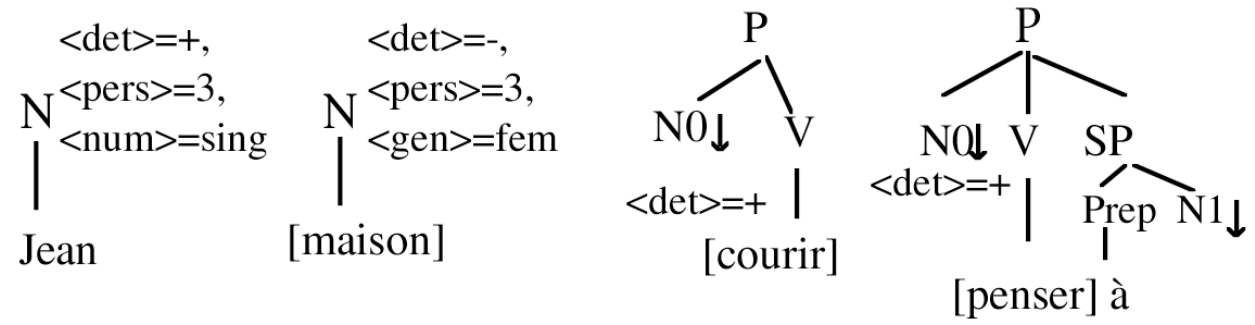

Arbres élémentaires initiaux:
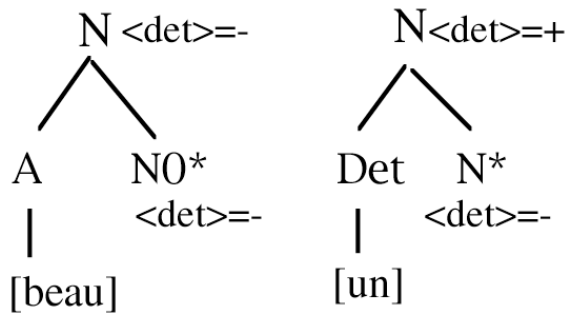

Arbres élémentaires auxiliaires d'autre part l'« arbre de dérivation », plus compact, qui indique quels arbres élémentaires ont été combinés à quels nœuds. La phrase Jean court vite, dérivée à partir des arbres élémentaires de la figure 2 , a les arbres dérivé et de dérivation suivants ${ }^{28}$ : 


\section{Arbre dérivé :}

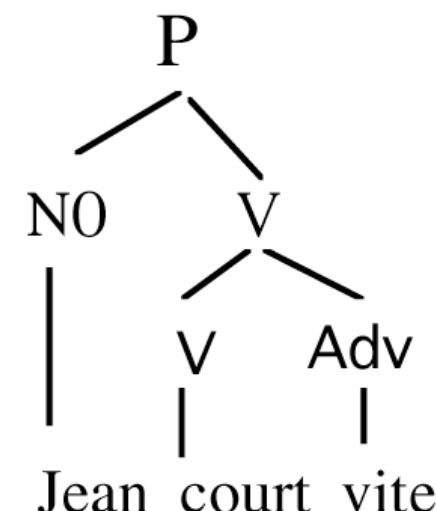

\section{Arbre de dérivation :}

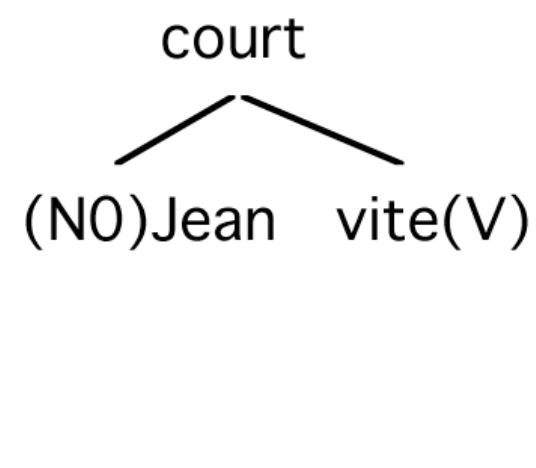

L'arbre initial de court a reçu la substitution de l'arbre de Jean à son nœud sujet (No) et l'adjonction de l'arbre de vite à son nœud interne (V). Par convention, dans un arbre de dérivation, l'arbre élémentaire domine les arbres qui ont été insérés par adjonction ou par substitution à ses nœuds. A partir du moment où chaque arbre élémentaire correspond à un mot (plein), l'arbre de dérivation est proche d'un arbre de dépendance sémantique.

Revenons maintenant aux verbes «à montée ». Les principes linguistiques qui régissent la bonne formation des arbres élémentaires sont généralement complétés de la façon suivante :

- le domaine de sous-catégorisation est étendu à la phrase : l'arbre élémentaire d'un prédicat a un nœud pour chacun de ses arguments sous-catégorisés réalisés, même dans une position non canonique. Ainsi, un complément extrait (Quel film Paul croit que Jean veut voir) est directement substitué à un nœud argumental dans l'arbre élémentaire étendu du verbe dont il dépend (ici voir), et les phrases enchâssantes intermédiaires sont introduites par adjonction $^{29}$.

- les positions correspondant à des arguments syntaxiques référentiels (les nœuds feuilles des arbres élémentaires prédicatifs) doivent recevoir un rôle sémantique du prédicat tête de l'arbre élémentaire, c'est-à-dire qu'on identifie tête syntaxique et tête sémantique ${ }^{30}$.

- un prédicat ne peut assigner directement un rôle sémantique qu'à ses arguments syntaxiques (c'est-à-dire aux nœuds de ses arbres élémentaires) ${ }^{31}$.

Dans ces conditions, on ne peut avoir de verbes «à montée " au sens des grammaires d'unification, c'est-à-dire de verbe (prédicatif) avec un sujet (ou un complément) syntaxique (référentiel) auquel il n'assignerait pas de rôle sémantique. On prévoit qu'on n'a que des auxiliaires (sans structure argumentale) ou des prédicats complexes (c'est-àdire des verbes fusionnant leurs arguments syntaxiques et sémantiques). L'analyse par prédicat complexe est adoptée en TAG pour les causatives, les verbes de perception et les constructions copulatives en français (cf. Abeillé (à paraître)). Je vais présenter ici l'analyse adoptée pour les auxiliaires de temps et les verbes «à montée » en français, et voir comment elle rend compte des propriétés mentionnées supra. 


\subsection{Les auxiliaires de temps adjoints au participe}

79 Je propose d'analyser l'auxiliaire temporel comme adjoint au participe passé, tête de l'arbre phrastique élémentaire (cf. Abeillé (1991)). On aura donc la dérivation suivante pour Jean a couru.

Figure 4. Dérivation de Jean a couru

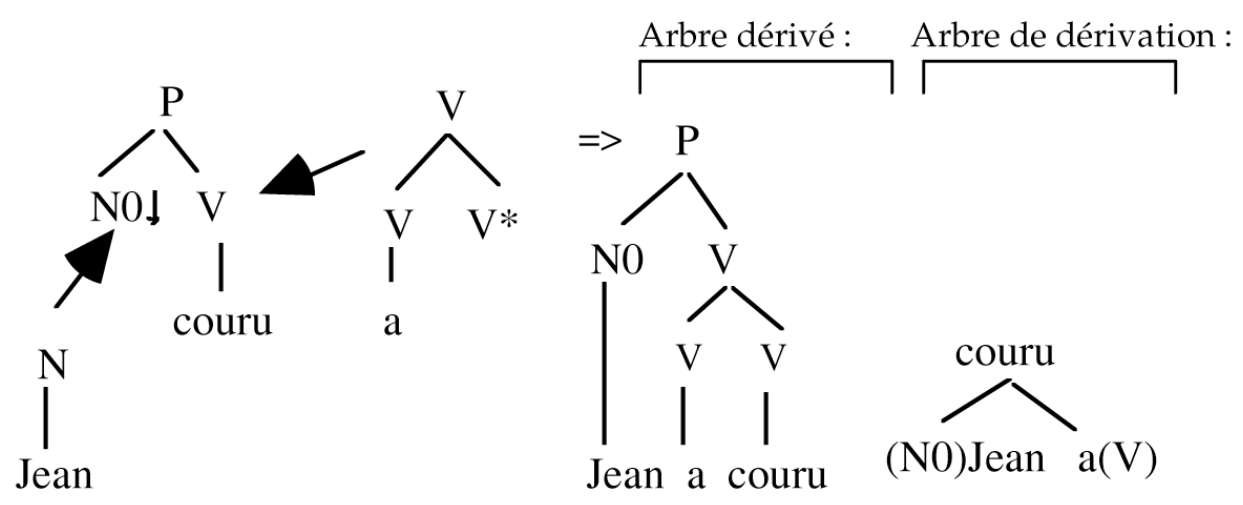

Dans cette analyse, le participe est la tête (syntaxique et sémantique) de la phrase, parce qu'il détermine la sous-catégorisation, et qu'il assigne aux arguments leur rôle sémantique. En TAG, ce ne sont pas les propriétés morpho-syntaxiques (accord, mode...) qui déterminent la tête syntaxique de l'arbre élémentaire. L'auxiliaire, qui n'apporte que ces marques morpho-syntaxiques, ainsi qu'une marque de temps (et d'aspect), ne peut donc être la tête de l'arbre phrastique. Il ancre un arbre élémentaire déficient (projection limitée à V). Le sujet est le sujet syntaxique et sémantique du participe, non de l'auxiliaire. Il est sélectionné entièrement par le participe. L'arbre de dérivation (figure 4) montre bien qu'il n'y a pas de dépendance entre sujet et auxiliaire.

81 Si l'on reprend les propriétés syntaxiques vues supra, il est clair que cette structure prédit directement que la séquence participe-compléments ne peut ni se cliver ni se pronominaliser, puisqu'elle n'est pas représentée comme formant un constituant. Aucune réalisation alternative du participe seul (clivage, pronominalisation) n'est par ailleurs disponible puisque celui-ci n'est pas un complément ${ }^{32}$.

Le placement des clitiques sur l'auxiliaire reçoit aussi une explication immédiate. En reprenant les arguments de Schane (1967), Kayne (1977), je suppose un sous-constituant verbal regroupant Verbe et Clitiques qui sont, en tant qu'arguments, substitués dans des arbres spécifiques dont est tête leur prédicat ${ }^{33}$. Le participe est la tête d'arbres élémentaires avec arguments clitiques comme n'importe quelle autre forme verbale. Le placement des clitiques se fait de la même façon avec le participe qu'avec une autre forme du même verbe. L'auxiliaire de temps s'adjoint sur le participe indépendamment de la présence des clitiques ${ }^{34}$ : 


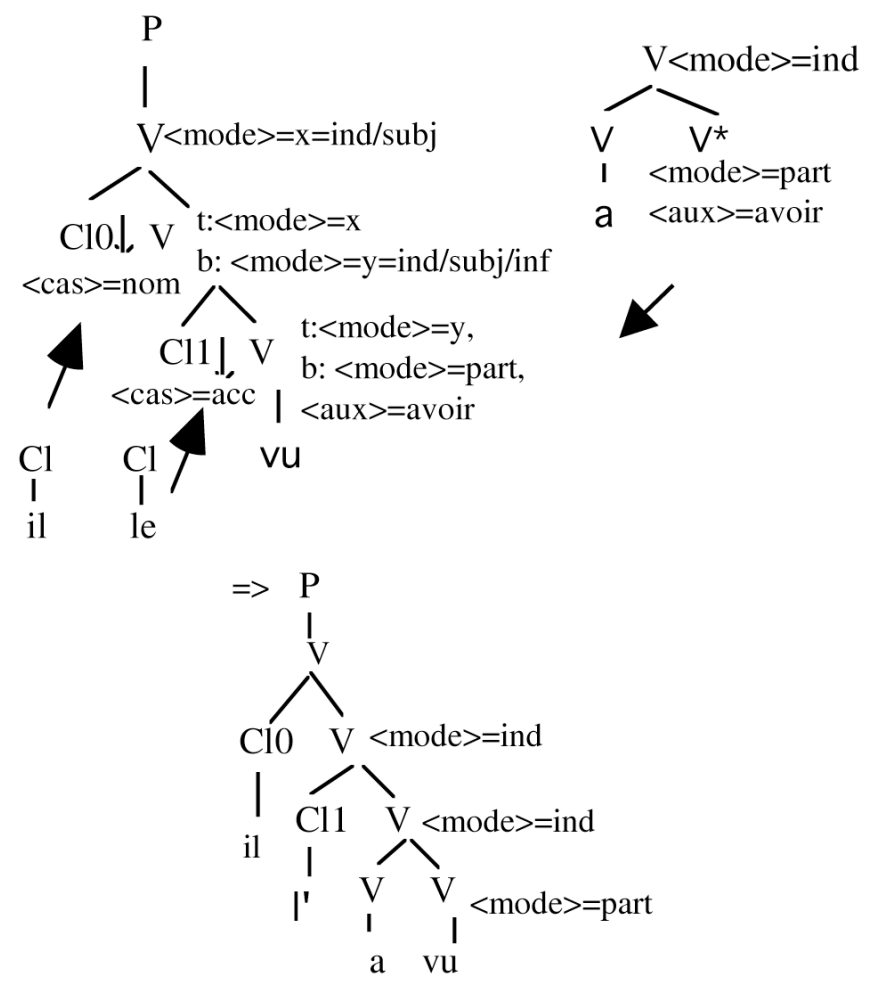

C'est une contrainte morphologique indépendante qui empêche l'adjacence du clitique et du participe $(*$ le $v u)$ et rend obligatoire l'adjonction de l'auxiliaire. Un verbe avec un clitique doit être à un mode fini (indicatif ou subjonctif avec un clitique sujet; indicatif, subjonctif ou infinitif avec un clitique complément). Or les nœuds $\mathrm{V}$ dominant les clitiques partagent nécessairement leur mode avec le nœud $\mathrm{V}$ du participe (variables $\mathrm{x}$ et y). Si la tête de l'arbre est un participe passé (comme figure 5), il y a conflit entre deux valeurs incompatibles pour le mode au nœud $\mathrm{V}$ inférieur. L'adjonction d'un auxiliaire est alors obligatoire car elle seule peut « séparer » les traits en conflit sur un même nœud ${ }^{35}$.

Reste la question des propriétés morpho-syntaxiques de l'auxiliaire, c'est-à-dire l'accord avec le sujet et la détermination du mode de la phrase. Elle sont prises en charge par les mêmes traits que ceux utilisés pour l'accord ou la détermination du mode pour les temps simples. Rappelons comment s'effectue la mise à jour des traits en cas d'adjonction: les traits du nœud qui reçoit l'adjonction doivent s'unifier avec ceux du nœud racine et avec ceux du nœud pied de l'arbre auxiliaire. Plus précisément, on distingue au nœud où a lieu l'adjonction des traits amont (ou top notés $\mathrm{t}$ ), qui doivent s'unifier avec ceux du nœud racine de l'arbre auxiliaire, et des traits aval (ou bottom notés b), qui doivent s'unifier avec ceux du nœud pied de l'arbre auxiliaire. Figure 6, l'équation d'accord avec le sujet, comme le partage du mode avec le nœud racine $\mathrm{P}$ se fait avec les traits amont du nœud V. En l'absence d'adjonction, traits amont et aval de $\mathrm{V}$ s'unifient entre eux et les traits de la tête verbale de l'arbre (qui doit être à un mode fini) sont normalement propagés. En cas d'adjonction, les traits amont de V sont unifiés avec ceux du nœud racine de l'arbre de l'auxiliaire (<mode> =indicatif) tandis que les traits aval sont « descendus » sous le nœud pied de l'arbre auxiliaire (<mode $>=$ participe). Ce sont donc les traits de l'auxiliaire qui sont pris en compte pour l'accord et la propagation du mode : 
Figure 6. Accord et propagation du mode

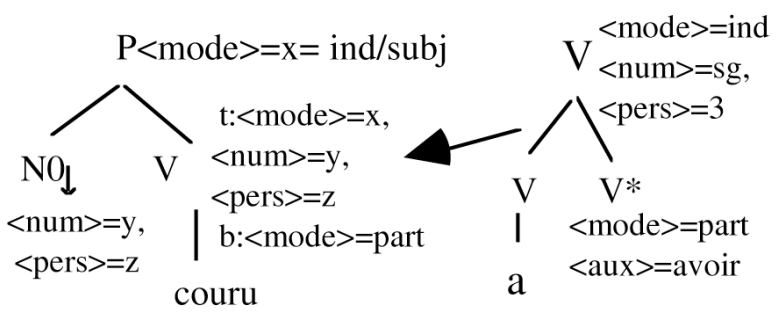

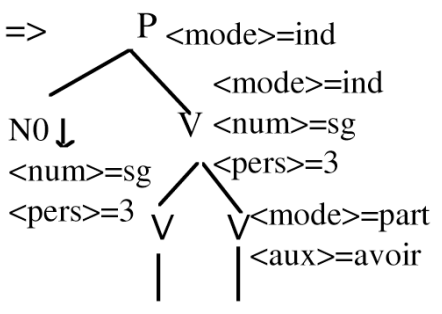

a couru

L'adjonction permet donc une « déviation » dans la propagation des traits « de tête »

\subsection{Les verbes « à montée » adjoints à l'infinitif}

Pour l'anglais, Kroch \& Joshi (1985) proposent une analyse par adjonction (à VP) des verbes "à montée», dans le cadre d'une grammaire TAG (non lexicalisée), sans développer ni l'interprétation ni la morpho-syntaxe associées ${ }^{37}$. En français, j'analyse les verbes dits "à montée " comme adjoints à l'infinitif, qui est la tête de la phrase, en ajoutant un trait pour le placement des clitiques ${ }^{38}$. Les arbres auxiliaires proposés sont par exemple les suivants :

Figure 7. Arbres auxiliaires pour verbes « à montée »
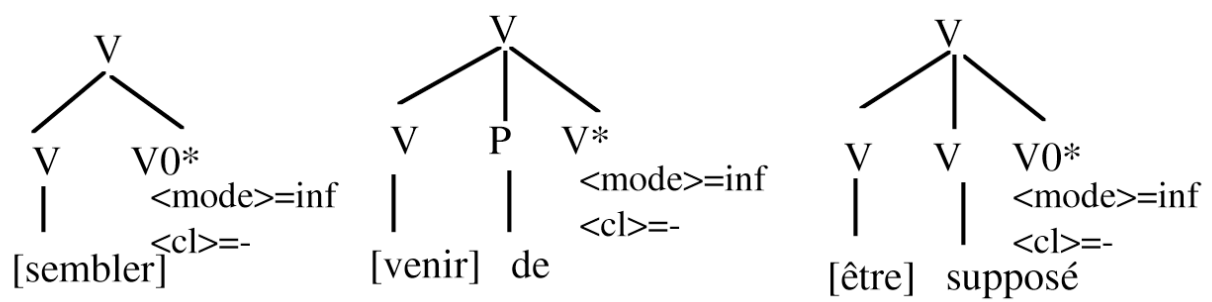

Ces structures sont proches de celles proposées pour les auxiliaires de temps. Si les verbes en question admettent d'autres constructions (un complément nominal pour les verbes aspectuels, une construction impersonnelle pour sembler ou paraitre), les structures correspondantes sont considérées comme indépendantes en syntaxe ${ }^{39}$.

L'analyse de la phrase Jean semble aimer Mozart est la suivante :

Figure 8. Dérivation pour Jean semble aimer Mozart

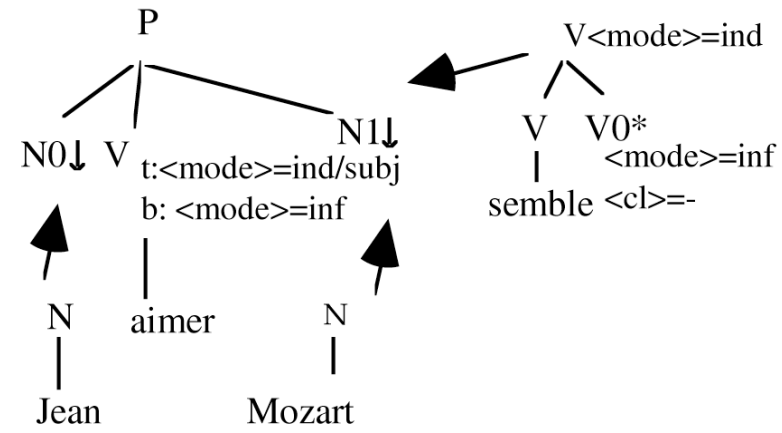

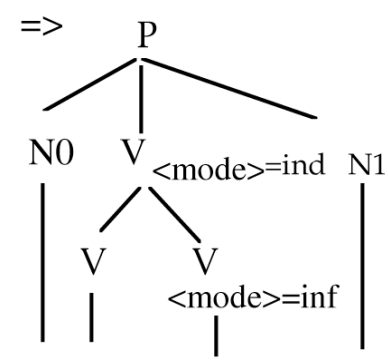

Jean semble aimer Mozart 

du verbe "à montée », qui a une projection limitée à V. L'infinitif, en tant que tête de l'arbre phrastique, sélectionne donc naturellement son sujet. Les sujets figés (ou idiomatiques) sont analysés comme co-têtes de l'arbre élémentaire du verbe dont ils dépendent (cf. Abeillé 1991). Dans le cas d'un verbe à sujet figé, comme pleuvoir, le sujet figé fait partie de l'arbre élémentaire du verbe. On aura bien il va pleuvoir par adjonction de va dans l'arbre de il pleuvoir:

Figure 9. Il semble pleuvoir

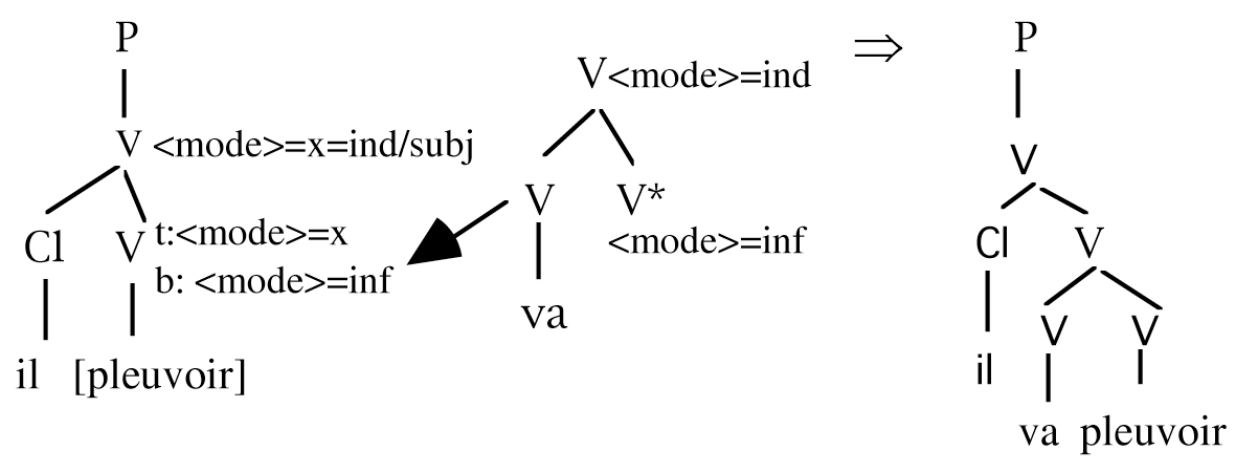

93 La phrase *Il veut pleuvoir n'est pas possible car il n'y a pas d'arbre autonome pour le il impersonnel qu'on pourrait substituer au nœud sujet de l'arbre élémentaire de veut.

\subsubsection{L'interprétation sémantique}

L'arbre de dérivation constitue en TAG la base de l'interprétation sémantique. Quelques arbres de dérivation pour les phrases « à montée » sont les suivants :

(a) Jean vient de voir Marie (b) Jean semble dormir (c) Il semble que Jean dort 
(a) Jean vient de voir Marie (b) Jean semble dormir (c) Il semble que Jean dort
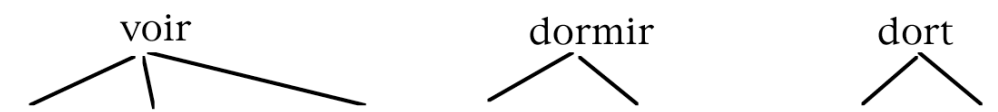

(N0)Jean vient-de(V) Marie(N1) (N0)Jean semble(V) (N0)Jean il-semble-que(P)

On peut noter que le sujet est, comme les compléments, sous la dépendance directe de l'infinitif. La contribution sémantique du verbe «à montée » est toujours représentée de la même façon, comme modifiant l'interprétation de l'infinitif, tête de l'arbre de dérivation. La prédiction est donc que la contribution sémantique du verbe "à montée " est de type adverbial, apportant des informations temporelles ou aspectuelles, et que l'infinitif est la tête sémantique de la phrase. C'est bien le cas pour la plupart des verbes « à montée ». Pour rendre compte des quelques verbes «à montée " prédicatifs (assignant un rôle sémantique à la proposition dénotée par l'infinitif), tels que sembler, être supposé..., il faut considérer que la dépendance sémantique est inversée (le verbe «à montée » est la tête sémantique de la phrase) ${ }^{41}$.

Quant à la relation entre Jean semble dormir et Il semble que Jean dort, il s'agit d'une paraphrase sémantique (sans lien syntaxique), comme le montrent les arbres de dérivation isomorphes (b) et (c) (avec il-semble-que comme arbre élémentaire à tête multiple s'adjoignant à la racine $\mathrm{P}$ ).

\subsubsection{Le placement des clitiques}

La différence entre auxiliaires de temps et verbes «à montée » en français pour le placement des clitiques compléments est prise en charge d'une part par une contrainte morphologique sur le mode des verbes à clitiques (qui empêche les clitiques d'être adjacents au participe), d'autre part par un trait $\langle\mathrm{cl}>$, qui note si un verbe a un argument cliticisé (et qui empêche l'adjonction d'un verbe "à montée " entre un clitique et l'infinitif). C'est ce qui empêche l'adjonction directe de peut sur voir figure 11. 
Figure 11. Dérivation pour Il peut le voir
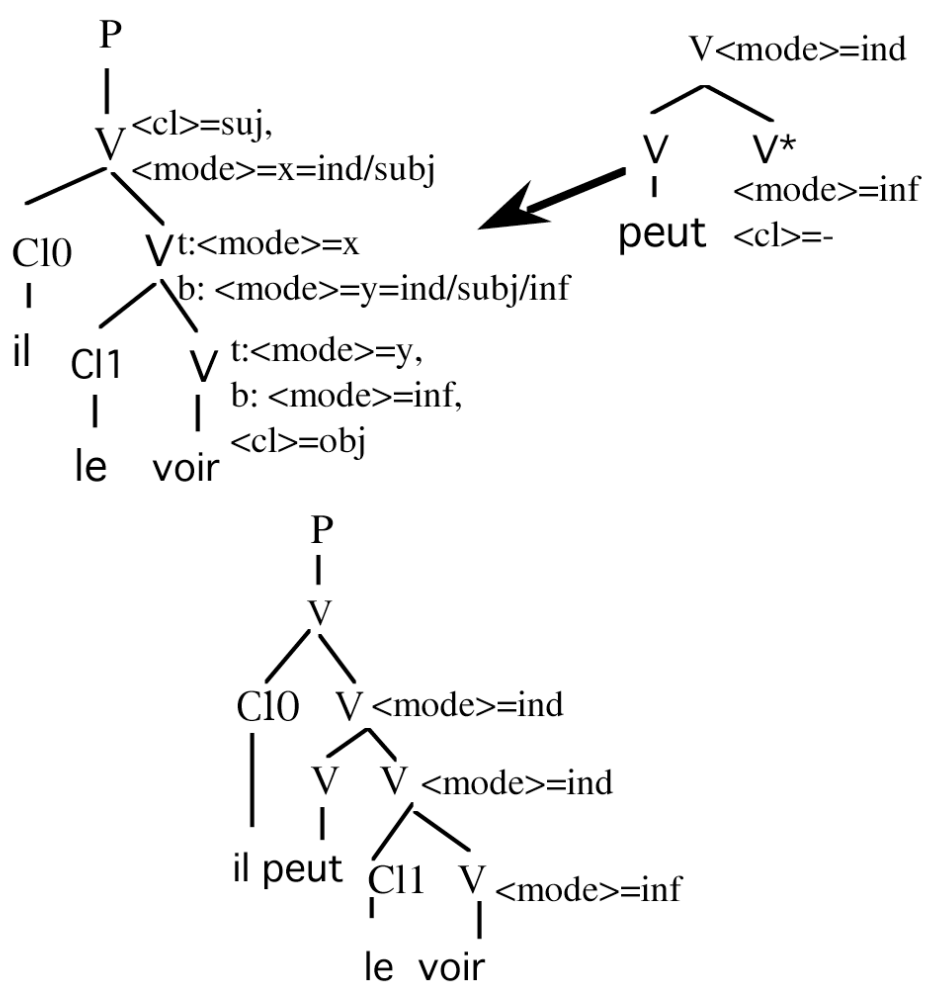

Les verbes "à montée » s'adjoignent entre le clitique sujet et les clitiques compléments (sauf en cas d'inversion où le verbe à montée s'adjoint sur le nœud $\mathrm{V}$ supérieur). La principale différence avec les auxiliaires de temps est donc une différence de trait: le verbe à montée contraint le trait $<\mathrm{cl}>\mathrm{du} \mathrm{V}$ sur lequel il s'adjoint tandis que l'auxiliaire ne le contraint pas $^{42}$. On aura donc bien le contraste attendu :

\begin{tabular}{|l|l|l|l|}
\hline & (59) & a. & Il peut le voir \\
\hline & & b. & *Il le peut voir \\
\hline
\end{tabular}

La conséquence pour les phrases à cliticisation du complément du sujet (cf. section 1 supra) est que le clitique en est à sa place habituelle de clitique complément (entre les verbes « à montée » et les auxiliaires de temps), et on a (60a) et non (60b) pour la même raison qu'on a (59a) et non (59b) ${ }^{43}$ :

\begin{tabular}{|l|l|l|l|}
\hline & $(60)$ & a. & Le bord semble en être déchiré \\
\hline & & b. & * Le bord en semble être déchiré \\
\hline
\end{tabular}

Aucune règle de mouvement n'est donc nécessaire pour expliquer la position de en.

Dans une grammaire TAG, on analyse donc les auxiliaires de temps et les verbes «à montée " comme adjoints. L'opération d'adjonction est mise à profit pour définir l'infinitif (ou le participe) comme tête à la fois syntaxique et sémantique de ces 
constructions. Cela permet de conserver (comme dans l'analyse transformationnelle) un principe de correspondance entre argument syntaxique et argument sémantique. Cette analyse rend compte immédiatement des propriétés communes à ces deux types de verbe (transparence vis-à-vis du sujet, tendance à la non prédicativité, déficience syntaxique). Pour les autres propriétés (placement des clitiques ou de la négation), la différence est prise en charge par des traits.

\section{Comparaison avec HPSG}

Les modèles TAG et HPSG ont en commun d'être « surfacistes » et basés sur des traits. Ils adoptent le « lexicalisme » c'est-à-dire le respect de l'intégrité lexicale en syntaxe ${ }^{44}$. Leurs différences sont connues: la grammaire syntagmatique guidée par les têtes (HPSG) se présente comme une théorie modulaire de la compétence linguistique, englobant phonétique, morphologie, syntaxe et sémantique, basée sur un formalisme mathématique peu contraignant. En TAG, on se limite généralement à la syntaxe, et le formalisme mathématique sous-jacent est plus contraint. Autre différence, les arbres élémentaires de TAG sont lexicalisés tandis que les règles syntagmatiques de HPSG peuvent avoir une tête non lexicale ${ }^{45}$.

Nous allons voir en quoi les analyses proposées pour les auxiliaires de temps en TAG et en HPSG sont convergentes, quoique différentes ; tandis que celles adoptées pour les verbes « à montée » sont incompatibles.

\subsection{L'auxiliaire de temps tête d'une structure « plate »}

Alors, comme on l'a vu, que l'essentiel des contraintes (et des prédictions) en TAG se place au niveau de l'interface syntaxe-sémantique, j'interprète les principes de HPSG comme mettant essentiellement l'accent sur l'interface syntaxe-morphologie. Une contrainte forte en HPSG est que la tête d'un syntagme soit sa tête morpho-syntaxique, conformément au Principe des traits de Tête, qui identifie la catégorie, le mode, le temps, etc. d'un syntagme à ceux de sa tête ${ }^{46}$. Le participe ne peut donc être la tête syntaxique de la phrase, bien que ce soit lui qui détermine la sous-catégorisation, car il ne peut pas être la tête morphologique d'une phrase finie ${ }^{47}$. A partir du moment où l'auxiliaire est la tête de la phrase, le sujet de la phrase doit être analysé comme le sujet syntaxique de l'auxiliaire (selon le Principe de Valence). Plusieurs généralisations en découlent immédiatement: les verbes s'accordent avec leur sujet syntaxique, seuls les verbes conjugués ont un sujet syntaxique réalisé.

C'est le point essentiel. Le second point, lui, est lié aux choix d'analyses effectués au sein du modèle, et non au modèle lui-même ${ }^{48}$. Pour l'anglais, Pollard \& Sag (1994), à la suite de Gazdar et al. (1982), analysent les auxiliaires anglais comme des verbes "à montée ", prenant un complément SV (participe ou infinitif), et de même pour les constructions copulatives (avec un complément syntagmatique de catégorie variable). C'est un trait AUX qui est chargé de rendre compte des propriétés morpho-syntaxiques spécifiques des auxiliaires anglais (négation, inversion, contraction).

Pour le français, nous avons rejeté ailleurs (Abeillé \& Godard (1996)) cette assimilation entre constructions «à montée ", temps composés et constructions copulatives. En nous appuyant notamment sur les propriétés rappelées supra, nous avons proposé une 
structure plate pour les temps composés ${ }^{49}$, où l'auxiliaire est la tête et hérite des compléments du participe qui est lui-même un de ses compléments ${ }^{50}$ :

Figure 12. Structures adoptées en HPSG
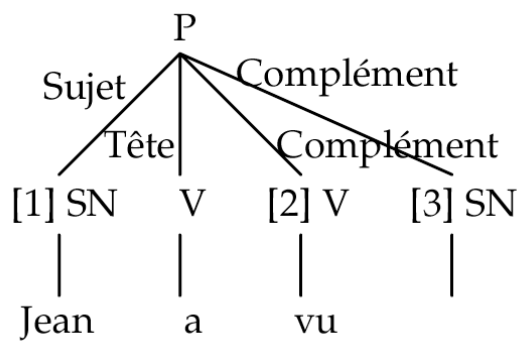

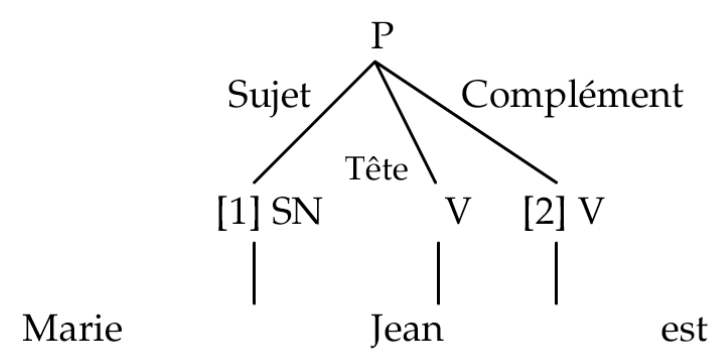

Sans entrer dans le détail de la description lexicale de l'auxiliaire (qui est sous-spécifiée puisque l'auxiliaire unifie la plupart de ses traits avec ceux du participe), l'entrée retenue pour $a$ est la suivante, une fois ses traits variables instanciés dans la phrase Jean a vu Marie :

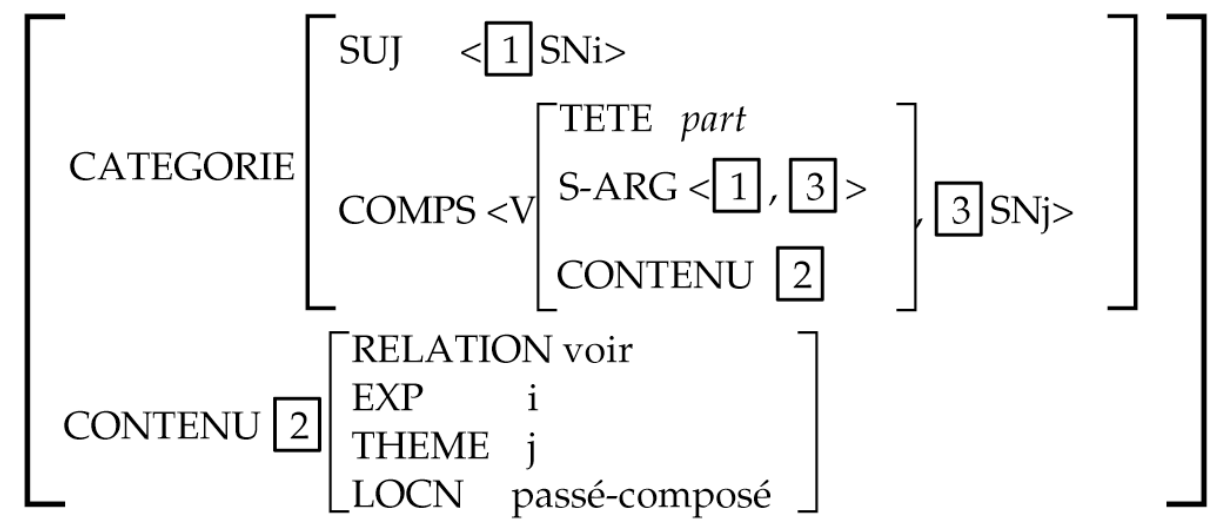

110 L'auxiliaire de temps attend un premier argument syntaxique (son sujet) identifié à celui du participe (), et une liste de deux compléments qui sont le participe et le SN () attendu par celui-ci. L'auxiliaire hérite du contenu du participe () auquel il ajoute une localisation aspecto-temporelle (trait LOCN). La coïncidence entre argument syntaxique et argument sémantique est respectée pour les arguments nominaux (en tant qu'il inclut dans son contenu le contenu du participe, l'auxiliaire assigne un rôle sémantique à son sujet (i) et à son complément nominal (j)) mais pas pour le participe qui est un complément sans rôle sémantique.

111 Le choix de la structure plate (avec le participe au même niveau que les compléments) là où TAG choisit une structure à complexe verbal, n'est pas vraiment discriminant. Dans les deux cas, on analyse la construction à temps composé comme une phrase simple (sans enchâssement syntagmatique) c'est-à-dire comme un seul domaine syntaxique et sémantique, et on rend compte des mêmes propriétés. Comme en TAG, le participe ne forme pas un constituant avec les compléments qui suivent, et ne peut être clivé ou pronominalisé avec eux. La réalisation des clitiques sur l'auxiliaire se fait sans mécanisme particulier puisque ceux-ci sont analysés comme compléments de l'auxiliaire. 
112 C'est le choix de l'auxiliaire comme tête (syntaxique et sémantique) qui est la principale différence avec l'analyse TAG. Mais il s'agit d'une tête au sens faible puisqu'elle hérite de la plupart des traits syntaxiques et sémantiques (sous-catégorisation, contenu...) du participe. Celui-ci est également un complément particulier, sans mobilité ni réalisation alternative possible, ce qu'on peut mettre au compte de sa «lexicalité » (ce n'est pas une projection syntagmatique), qui le rapproche d'autres compléments «lexicaux » tels que les noms sans déterminant après les verbes supports : avoir faim, rendre hommage (cf. Abeillé \& Godard (1996)).

\subsection{Les verbes « à montée » têtes d'une structure hiérarchique}

113 Le même raisonnement que ci-dessus s'applique aux verbes à montée : l'infinitif ne peut être la tête de la phrase, laquelle ne peut être que le verbe " à montée ", puisque c'est lui qui détermine le mode de la phrase. Le sujet de la phrase est donc le sujet syntaxique du verbe « à montée ».

114 Par ailleurs, on considère, en HPSG, comme en LFG (Bresnan 1982) ou en GPSG, que les verbes « à montée », comme les verbes à contrôle, prennent un complément SV :

Figure 13. Montée et contrôle en HPSG

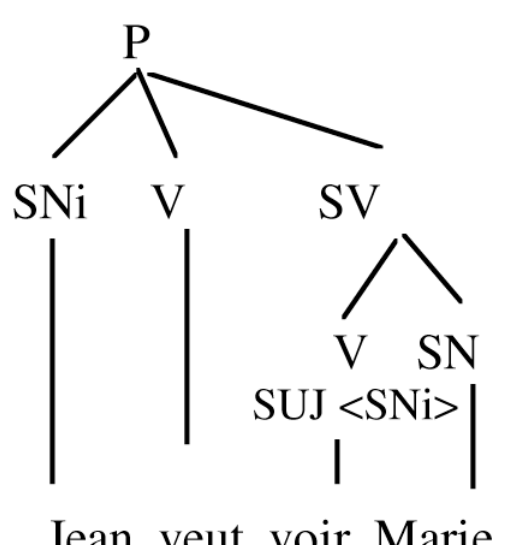

Jean veut voir Marie

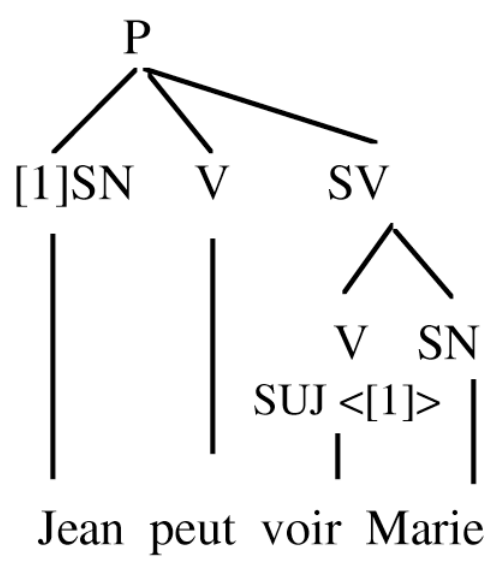

Cette structure n'a pas été remise en cause pour le français (cf. Miller 1991, Baschung 1992, 1998, Abeillé \& Godard 1996). Cela revient à dire que les propriétés retenues comme discriminantes pour la structure syntaxique sont le placement des clitiques et de la négation: avec une analyse morphologique de la cliticisation (considérée comme affixation), le placement des clitiques est forcément local (sur le verbe dont ils sont un complément), et le complément SV (dont la tête est l'infinitif) doit contenir tous ses clitiques. De la même façon, si l'on analyse ne-pas comme adjoint à SV (infinitif), et non à $\mathrm{V}$, on explique la possibilité de la négation après verbe à montée et son impossibilité après l'auxiliaire.

Quelle différence reste-t-il entre montée et contrôle? Selon Pollard \& Sag (1994), l'identification des sujets se fait par partage intégral des traits (noté [1] figure 13) pour la construction "à montée ", tandis qu'elle se fait par coïndiciation (indice i) pour la construction «à contrôle». Les verbes à contrôle attendent donc un sujet référentiel (auquel ils assignent un rôle sémantique) tandis que les verbes à montée peuvent prendre n'importe quel sujet attendu par l'infinitif. A la différence de l'analyse des auxiliaires de 
temps présentée ci-dessus, c'est ici le sujet qui est un argument syntaxique sans être un argument sémantique ; le seul argument sémantique est celui dénoté par le complément verbal. L'entrée de sembler est donc la suivante (simplifiée) :

$117(62)$

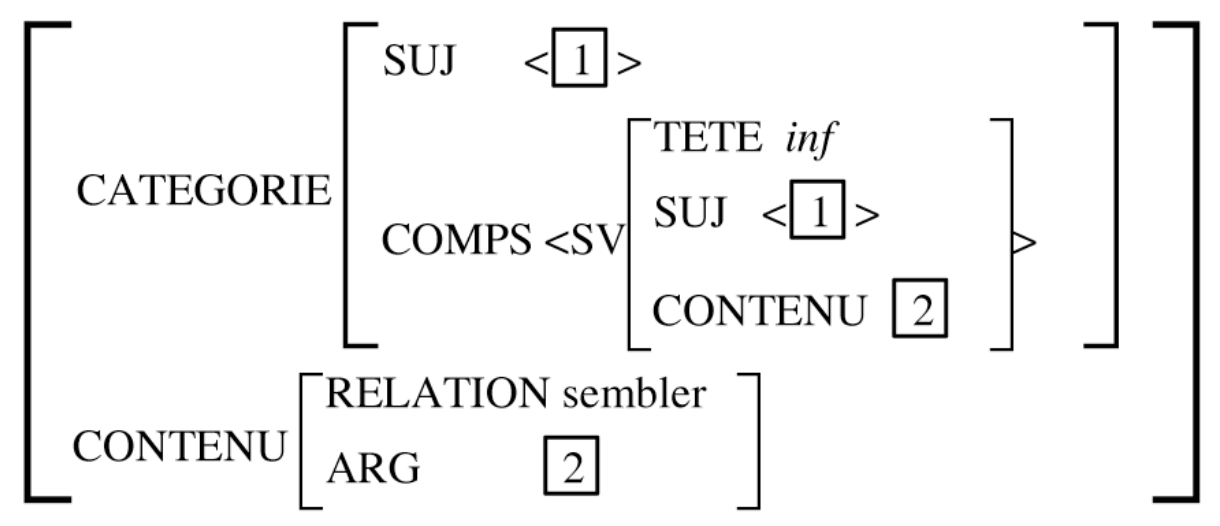

118 Afin de rendre compte de la "déficience» syntaxique des verbes «à montée » (propriétés II supra), on peut ajouter un trait distinctif, en déclarant le complément infinitif des verbes "à montée » comme étant de type "canonique ", ce qui l'empêche d'être extrait ou cliticisé (le complément des verbes à contrôle n'ayant pas cette restriction $)^{51}$. Une autre solution est de s'appuyer sur un "principe de montée » plus général, défini ainsi par Pollard \& Sag (1994) :

119 Un verbe n'est autorisé à ne pas assigner de rôle sémantique à un de ses arguments (non explétif) que dans la mesure où il sous-catégorise également un complément non saturé dont le sujet est identifié avec cet argument ${ }^{52}$.

Ce principe a d'abord pour tâche de limiter les cas de distorsion entre argument syntaxique et argument sémantique aux verbes à complément verbal ou attributif (ce qui inclut les constructions copulatives) ${ }^{53}$. Mais il permet aussi de rendre compte de la déficience syntaxique des verbes "à montée " prédicatif $^{54}$ : en cas d'extraction ou de pronominalisation, le verbe "à montée » ne prend plus de complément SV non saturé (il est marqué comme ayant un argument SV «extrait» ou prend un complément pronominal saturé). Ainsi : * La pluie le doit ou * Ce que la pluie commence (c'est à tomber) sont exclus car la pluie ne reçoit pas de rôle sémantique de doit ou commence sans être coïndiciée au sujet d'un complément infinitif non saturé, ce qui est exclu en vertu du « principe de montée $»^{55}$.

\section{Conclusion}

121 J'ai tenté de montrer qu'à côté des propriétés bien connues des auxiliaires et des verbes « à montée » en français (c'est-à-dire la transparence vis-à-vis du sujet et la tendance à la non prédicativité), il fallait ajouter certaines déficiences syntaxiques (la séquence verbale qui suit l'auxiliaire ou le verbe "à montée » n'a pas toutes les propriétés attendues d'un complément) et j'ai voulu montrer comment ces propriétés peuvent être interprétées dans le cadre de deux théories syntaxiques récentes qui travaillent uniquement sur les structures de surface. 


\section{BIBLIOGRAPHIE}

ABEILLE, A. (1991). Une grammaire lexicalisée d'arbres adjoints pour le français. : application à l'analyse automatique Thèse de Doctorat, Université Paris 7. (édition revue à paraître aux éditions du CNRS)

ABEILLE, A. (1993). Les nouvelles syntaxes : grammaires d'unification et analyse du français, Paris : Armand Colin.

ABEILLE, A. (1996), Fonction ou position objet? $1^{\text {ère }}$ partie, Gré des Langues 11, pp. 8-29. pp. 32-61. 
ABEILLE, A., GODARD, D., MILLER P (1997) Les constructions causatives en français : un cas de compétition syntaxique, Langue française, 115, pp. 62-74.

ABEILLE A. (1998) Extraction out of NP and clitic-noun dependencies in French, in Abeillé \& Rambow (eds) Tree Adjoining grammars, Stanford: CSLI (à paraître).

ANDREWS, A. (1982) The representation of case in modern Icelandic, in Bresnan (ed) The mental representation of grammatical relations, Cambridge: MIT Press.

BASCHUNG, K. (1992) Grammaires d'unification à traits et contrôle des infinitives en français, Clermont Ferrand : Adosa.

BASCHUNG, K. (1998) Le contrôle revisité : y a-t-il une différence entre verbes 'à contrôle' et verbes 'à montée' ? Journal of French Language Studies, 8 :1, pp. 1-27.

BLANCHE-BENVENISTE, C., DELOFEU J., STEFANINI J., Van den EYNDE K. (1987) Pronom et syntaxe : l'approche pronominale et son application au français Paris : Selaf. (2e édition)

BLANCHE-BENVENISTE, C., BILGER M., ROUGET C., Van den EYNDE K. (1991) Le Français Parlé Etudes Grammaticales, Paris : Editions du CNRS.

BRATT, E. (1990) The French causative construction, ms, Stanford University.

BRESNAN, J. (1982) Complementation and control, in Bresnan (ed) The mental representation of grammatical relations, Cambridge: MIT Press.

CANDITO, M.-H. (1996) A principle-based and hierarchical representation of LTAGs. Actes 15ème COLING, Copenhagen.

CANDITO, M.-H. (1999) Une organisation modulaire et paramétrable de grammaires lexicalisées : application au français et à l'italien, Thèse de doctorat, Université Paris 7.

CANDITO, M.-H., KAHANE S. (1998) Une grammaire TAG vue comme une grammaire sens-texte précompilée. Actes 5ème TALN, Paris.

CHOMSKY, N. (1973) Conditions on transformations, in S. Anderson, P. Kirsparsky (eds), A Festschrift for M. Halle, New York: Holt, Reinhart, Winston.

CHOMSKY, N. (1981) Lectures on Government and Binding, Dordrecht: Foris.

CHOMSKY, N. (1986) Barriers, Cambridge: MIT Press.

COUQUAUX, D. (1980) Place de la transformation «montée » dans la syntaxe du français moderne, Le Français Moderne, 48, pp. 193-218.

DOMINICY, M. (1979) Compte rendu de M. Gross : Méthodes en Syntaxe, Revue Romane, 14, pp. 293-318.

EMONDS, J. (1978) The verbal complex V’ V in French, Linguistic Inquiry, 9-2, pp. 151-175.

FRANK, R. (1992) Syntactic locality and Tree Adjoining grammar: grammatical, acquisition and processing perspectives, Thèse de $\mathrm{PhD}$, University of Pennsylvania, Philadelphia.

FUCHS, C., LE GOFFIC P. (1992) Les linguistiques contemporaines, Paris : Hachette.

GAZDAR, G., PULLUM G., I. SAG (1982) Auxiliaries and related phenomena in a restricted theory of grammar, Language, 58, pp. 591-638.

GREVISSE, M. (1988) Le Bon usage, Louvain : Duculot (12e édition revue par A. Goosse).

GROSS, M. (1975) Méthodes en syntaxe, Paris : Hermann.

HUOT, H. (1974) Le verbe devoir : étude synchronique et diachronique, Paris : Klincksieck. 
JACOBSON, P. (1990) Raising as Functional composition, Linguistics and Philosophy, 13, pp. 423-475.

KAYNE, R. (1974-75) French Relative que, Recherches Linguistiques de Vincennes, II, pp. 40-61, III, pp. 27-92.

KAYNE, R. (1977) Syntaxe du français : le cycle transformationnel, Paris : Seuil.

KAYNE, R. (1980) De certaines différences entre le français et l'anglais, Langages, 60, pp. 47-64.

KROCH, A., JOSHI A. (1985) The linguistic relevance of Tree Adjoining Grammars, Technical report 85-16, University of Pennsylvania, Philadelphia.

LANGACKER, R. (1995) Raising and transparency, Language 71, pp. 1-62.

MILLER, P. (1991) Clitics and constituents in phrase structure grammar, Thèse de PhD, Université d'Utrecht. (publiée 1992, New York: Garland)

MILLER, P., SAG I. (1997) French clitic movement without clitic or movement, Natural Language and Linguistic Theory.15, pp. 573-639.

MONACHESI, P. (1995) A grammar of Italian Clitics, Thèse de PhD, Tilburg. (à paraître Stanford : CSLI Dissertations series)

NUNBERG, G., SAG I., WASOW T. (1994) Idioms, Language , 70-3, pp. 491-538.

PERLMUTTER, D. (1970) The two verbs begin in Jacobs \& Rosenbaum (eds) Readings in English Transformational grammar, Waltham: Ginn, pp. 107-119.

POLLARD, C., SAG I. (1994) Head-driven Phrase Structure Grammar, Chicago: University of Chicago Press.

POLLOCK, J.-Y. (1989) Verb Movement, Universal Grammar and the Structure of IP, Linguistic Inquiry, 20, pp. 365-424.

RIZZI, L. (1978) A restructuring rule in Italian, in Keyser (ed) Recent transformational studies in European languages, Cambridge: MIT Press, pp. 113-158.

ROCHETTE, A. (1988) Semantic and syntactic aspects of Romance sentential complementation, Thèse de PhD, MIT.

ROORYCK, J. (1989) Les verbes à montée et à contrôle " ambigus ", Revue Québécoise de Linguistique, 18 :1. pp. 189-206.

ROORYCK, J. (1990) Montée et contrôle : une nouvelle analyse ?, Le Français Moderne, 58, pp. 1-27.

ROSENBAUM, P. (1967) The grammar of English predicate complement construction, Cambridge: MIT Press.

RUWET, N. (1972) En et la transformation Montée, in Théorie syntaxique et Syntaxe du français, Paris : Seuil.

RUWET, N. (1983) Montée et contrôle : une question à revoir, in Mélanges à Carl Vikner, M. Herslund et al. (eds), Etudes Romanes, Copenhagen: Akademisk Forlag.

RUWET, N. (1991) Syntax and Human experience, Chicago: University of Chicago Press.

SAG I., KARTTUNEN L., GOLBERG J. (1992) A lexical analysis of Icelandic case, in Sag \& Szabolcsi (eds) Lexical matters, Stanford: CSLI.

SCHANE S. (1967) La phonologie du groupe verbal en français, Langages, 7.

SUEUR, J-P. (1979) Analyse sémantique des verbes devoir et pouvoir, Le Français Moderne, 47-2, pp. 97-119. 
TASMOWSKI, L. (1980) Un devoir opérateur, Travaux de linguistique, 7, pp. 43-58.

TASMOWSKI, L. (1990) En semble... ou semble en..., Le Français Moderne, 58, pp. 28-42.

VAN EYNDE F. (1994) Auxiliaries and verbal affixes : a monostratal crosslinguistic analysis, Thèse de PhD, Université Catholique de Louvain.

WILLEMS, D. (1969) Analyse des critères d'auxiliarité en français moderne, Travaux de Linguistique, 1, pp. 87-98.

ZRIBI-HERTZ, A. (1986) Relations anaphoriques en français Esquisse d'une grammaire générative raisonnée de la réflexivité et de l'ellipse structurale, vol. 2, Thèse de Doctorat d'Etat, Université Paris 8.

ZWICKY, A. (1978) Arguing for constituents, Papers of the 14th regional meeting of the Chicago Linguistic Society, Chicago.

ZWICKY, A. (1985) On heads, Journal of Linguistics, 21:1, pp. 1-30.

\section{NOTES}

1. Je voudrais remercier pour leurs jugements ou pour leurs commentaires: P. Amsili, K. Baschung, M. Candito, P. Caudal, L. Clément, D. Godard, J. Jayez, S. Kahane, L. Roussarie ainsi qu'un lecteur anonyme de LINX.

2. En dehors de la tradition générative, la dénomination des verbes "transparents " varie selon les auteurs : verbes « auxiliaires» (Willems 1969), verbes «opérateurs» (Gross 1975), verbes « semi-auxiliaires» (Grevisse 1988), verbes « sans valence » (Blanche-Benveniste et al. 1991).

3. Il faut distinguer absence de contrainte sur le sujet et transparence. Les verbes psychologiques, par exemple, ne contraignent pas leur sujet - qui peut être agentif ou non, nominal ou phrastique (cf. Gross (1975)) :

Jean / Le vent / Qu'il pleuve épouvante Marie

Mais ces verbes assignent un rôle sémantique à leur sujet - qui doit être référentiel. Ils ne sont pas transparents.

4. Les difficultés ont été surtout soulignées pour les verbes "à montée du sujet en position objet », ou «transparents » pour leur objet (believe, expect... en anglais ; laisser, voir... en français). La transformation ayant été abandonnée depuis Chomsky (1973), l'analyse à complément phrastique (believe (John to be a liar)) doit ajouter la possibilité d'une assignation de cas exceptionnelle (par believe au sujet enchassé) et un passif exceptionnel en anglais (John is believed to be a liar). L'analyse surfaciste à deux compléments (believe (John) (to be a liar)) est évidemment plus simple. Faute de place, je ne discuterai pas l'équivalent français de ces constructions dans cet article.

5. Si l'on abandonne les transformations ordonnées, l'explication de l'agrammaticalité de (10)c, proposée par Couquaux (1980) dans un cadre GB, est que en (sur semble) ne peut plus lier la catégorie vide correspondant au complément prépositionnel (resté dans l'infinitive). Pour expliquer (10)b, Couquaux doit supposer que le sujet «monte » en laissant la catégorie vide de son complément dans l'infinitive (ainsi liée par en), c'est-à-dire une opération de scission ad hoc entre nom et complément de nom (cf. Tasmowski (1990)).

6. Pour Tasmowski (1990), seuls les verbes « inaccusatifs » (sans passif impersonnel) autorisent la cliticisation du complément $d u$ sujet. Cette dernière serait analogue à la cliticisation du complément d'un objet profond, et le placement de en en (10)b recevrait la même explication qu'en (ii) : 
(i) Paul semble aimer le goût des épinards

(ii) Paul semble en aimer le goût

7. Sans vouloir affirmer que le détail des sous-catégorisations syntaxiques est entièrement prédit par la sémantique lexicale, il semble naturel de penser que celle-ci contraint le nombre (maximal) d'arguments syntaxiques, voire leur catégorie. On explique ainsi que, dans toutes les langues, un prédicat signifiant dormir (qui n'a qu'un argument sémantique) n'a qu'un argument syntaxique, tandis qu'un prédicat signifiant frapper en a deux. Le nombre d'arguments syntaxiques effectivement réalisés pour un prédicat donné peut bien sûr être inférieur à son nombre d'arguments sémantiques (par exemple en cas d'ellipse), mais on voit mal comment il pourrait lui être supérieur (sauf arguments syntaxiques figés ou explétifs).

8. Sa conclusion rejoint celle de Ruwet $(1983,1991)$ qui propose aussi un continuum entre constructions "à montée » et constructions "à contrôle", les propriétés caractéristiques des premières étant corrélées au caractère non agentif du sujet et à l'aspect non ponctuel du procès dénoté par l'infinitif

9. On observe des variations en diachronie comme en synchronie concernant la liste exacte des verbes suivis d'un infinitif et «transparents » vis-à-vis de leur sujet. La plupart des locuteurs ont en commun les modaux (pouvoir, devoir), les temporels venir de et aller, les aspectuels (cesser de, commencer à, se mettre à, être en train de, finir de...), différents prédicats (sembler, paraître, s'avérer, risquer de, avoir failli, menacer, promettre) et quelques locutions (avoir beau, être fichu de, être susceptible de, avoir coutume de, avoir tendance à, être loin de). On peut y ajouter des passifs transparents soit figés (être censé) soit sans lien syntaxique avec l'actif correspondant (être garanti, être supposé, être dit ...).

10. Le supposé auxiliaire être au passif, que nous analysons en fait comme la copule être, a des propriétés différentes de celles des auxiliaires de temps (cf. Abeillé \& Godard (1996)). Je l'ignore ici.

11. Dominicy (1979), qui critique Gross, cite Signoret (1976, La nostalgie n'est plus ce qu'elle était, p. 351) : «Ils sont en train de ne pas manger ». Nous avons nous-même relevé (Fuchs \& Le Goffic (1992), p. 82) : «Les transformations, qui continuent à ne pas engendrer de structures lexicales, sont définies ...»

12. Comme Baschung (1998), je pense que certaines propriétés mentionnées dans la littérature ne fournissent pas de résultats concluants : par exemple, l'existence (ou la non existence) d'une complémentation alternative où un SN ou un SP «remplace" l'infinitive, ne dit rien de la construction de l'infinitive. L'antéposition de la séquence verbale, impossible avec l'auxiliaire (* $B u d u$ vin, il a), parfois possible avec un verbe à montée ou à contrôle (Rédiger sa thèse, il est en train / il voudrait bien), est soumise à des contraintes sémantiques mal connues et rien ne dit que le lien entre la séquence antéposée et le verbe principal soit un lien syntaxique plutôt que sémantique.

13. Les verbes restructurants en italien " perdent » de la même façon tout autre complément que l'infinitive.

14. La seule exception (pour certains locuteurs) est le complément datif du verbe sembler ; celuici est moins bon avec l'infinitive qu'avec l'adjectif attribut, et l'on peut considérer que la construction adjectivale est une construction copulative différente de la construction «à montée ».

(i) Paul semblait endormi à ses parents

(ii) Paul leur semblait endormi

(iii) ? ? Paul semblait dormir à ses parents

Avec l'infinitive, il est mieux accepté sous forme de pronom faible et peut être considéré comme « incorporé » :

(iv) Paul leur semblait dormir

15. Les clivées sont difficiles avec les complétives ou les infinitives, c'est pourquoi nous leur préférons le test des pseudo-clivées. 
16. Semblable à risquer est le cas d'avoir failli, que Blanche-Benveniste et al. (1991) proposent d'analyser comme ambigu (action manquée ou non accomplissement). On a bien le contraste :

* Ce qu'il a bien failli, c'est pleuvoir.

? Ce que Jean a bien failli, c'est démissionner.

17. On préfère généralement l'ellipse du complément:

mais sa sœur commencera demain / va continuer pendant tout l'été

18. Le clitique est accepté avec sembler par certains locuteurs ; mais le contraste est clair avec la construction copulative (où il est accepté par tous) :

Les rosiers semblent bien taillés mais les azalées ne le semblent pas

19. Comme le note Kayne (1977, p. 247), cette correspondance n'est pas absolue, puisqu'on peut avoir l'interprétation "déontique" avec certains sujets non référentiels: Assistance doit être portée aux personnes en danger. Mais une bonne dizaine d'autres tests ont été présentés pour distinguer les deux emplois dans la littérature.

20. Ce tour est semblable au "mouvement long de $\mathrm{SN}$ » rencontré en italien avec les verbes restructurants. Dans ce cas, c'est l'équivalent du moyen qui se réalise sur le verbe restructurant ( possere) :

Queste case si possono vendere a caro prezzo (lit. Ces maisons se peuvent vendre cher)

21. Comme me l'a suggéré D. Godard, la morphologie passive doit exister indépendamment de l'emploi "à montée", ce qui explique que seuls les aspectuels (qui ont par ailleurs un complément SN passivable) soient possibles ici.

22. Les verbes «à montée » français sont donc plus proches qu'il n'y paraît de leurs homologues des autres langues romanes. Ils en ont perdu la propriété la plus frappante (montée des clitiques) mais ils gardent néanmoins certaines propriétés de verbes "restructurants " (déficience syntaxique de l'infinitive, tour à Inf, montée du passif). On peut penser que ces propriétés sont résiduelles et que l'évolution qui les assimile (depuis le 18ème siècle) aux verbes à contrôle n'est pas encore achevée en français, ou bien que c'est leur déficience sémantique (non prédicativité ou transparence), proche de celle des auxiliaires, qui empêche leur assimilation syntaxique complète aux verbes à contrôle.

23. La contradiction n'éclate que pour le français. En italien, les verbes «à montée » (en tant que verbes restructurants) ont aussi des propriétés contradictoires, mais chaque bloc de propriétés est corrélé au placement des clitiques compléments : analyse comme auxiliaires (et clitiques en haut) ou analyse comme verbes à complément syntagmatique (et clitiques sur l'infinitif) (cf. Rizzi (1978) ou Monachesi (1995)).

24. L'analyse transformationnelle, qui a d'autres inconvénients (cf. supra), peut accommoder cette situation en définissant trois catégories différentes de compléments: VP pour les auxiliaires de temps, S (ou IP) pour les verbes à montée et $\mathrm{S}^{\prime}(\mathrm{ou} \mathrm{CP})$ pour les verbes à contrôle (cf. Rochette 1988, Pollock 1989).

25. ences citées.

26. Ils codent à la fois la sous-catégorisation, les réalisations syntaxiques et l'ordre des mots. Dans une optique plus modulaire, on peut les voir comme le résultat du croisement de descriptions partielles codant séparément ces différents types d'informations (Candito 1996, 1999).

27. Le signe 哃 marque les nœuds à substitution, et le signe ${ }^{*}$ les nœuds pieds. Les crochets [courir] indiquent qu'on peut avoir n'importe quelle forme fléchie du lemme correspondant, et les indices $(0,1)$ que le nœud correspond à un argument sémantique. Ces arbres sont simplifiés

28. Dans l'arbre de dérivation, je note ici pour simplifier la tête lexicale (et non le nom) des nœuds où ont lieu les combinaisons. 
29. Une conséquence est que les structures élémentaires ne sont pas toujours des arbres au sens strict, notamment pour celles qui ont pour tête un nom dont un complément est extrait hors du SN.

30. Dans les expressions idiomatiques, on peut avoir un argument syntaxique (non référentiel) sans rôle sémantique, par exemple un complément figé passivable comme dans déterrer la hâche de guerre. Il correspond à un nœud dominant une co-tête lexicale et non à un nœud feuille.

31. On considère généralement, pour les constructions à contrôle, que l'infinitif peut ancrer un arbre phrastique à sujet vide auquel il assigne un rôle sémantique. On doit ajouter un module de coréférence identifiant l'argument vide à son contrôleur.

32. Dans une grammaire TAG, les constructions clivées, comme les autres phénomènes d'extraction, sont représentées par des arbres élémentaires alternatifs à tête verbale (pour chaque argument correspondant à un nœud occupé par une catégorie syntagmatique dans un arbre à racine phrastique).

33. Un traitement des clitiques comme affixes verbaux (suivant Miller (1991) et Miller \& Sag (1997)) est aussi possible en TAG. Je ne le retiens pas afin de simplifier l'interface avec la morphologie (segmentation) et l'interface avec la sémantique (dérivations isomorphes avec pronoms clitiques - Paul lui ressemble - et pronoms forts - Paul pense à lui).

34. En reprenant notamment les données de Miller (1991), je considère que les clitiques sujets (qui peuvent avoir portée large sur deux verbes coordonnés) sont plus hauts dans l'arbre que les clitiques compléments (qui ne le peuvent pas) :

(i) Il l'admirait et l'observait

(ii) * Il l'admire et observe

35. La même contrainte morphologique empêche l'auxiliaire de s'adjoindre sur le nœud V supérieur (* a le vu). Voir par ailleurs Abeillé (1991) pour la sélection de l'auxiliaire (trait <aux>) et Candito (1999) pour l'accord du participe passé, que j'ignore ici.

36. L'adjonction force à opter pour une structure à complexe verbal (cf. Emonds 1978 et structure B supra). Une autre possibilité serait de définir une structure plate (comme C supra) dont le participe serait la tête, précédée d'un nœud où serait substitué l'auxiliaire. Cette analyse aurait l'inconvénient de ne pas associer les mêmes arbres élémentaires aux participes et aux autres formes verbales et de ne pas rendre compte des "surcomposés » : quand Jean a eu fini... C'est pourquoi je ne l'ai pas retenue.

37. Dans une optique où les arbres élémentaires comportent des nœuds pour les têtes fonctionnelles (I, C), Frank (1992), s'inspirant de l'analyse adoptée en GB, propose une analyse par adjonction à IP en développant la propagation du trait de cas : l'infinitif ne pouvant assigner de cas nominatif à son sujet, l'adjonction d'un verbe à montée est obligatoire ; cette analyse est en contradiction avec les faits de l'islandais (mentionnés supra) et ne résout ni la question de l'accord du sujet ni celle de la propagation du mode.

38. Cette analyse rejoint la caractérisation par Blanche-Benveniste et al. $(1987,1991)$ des verbes « à montée » comme verbes «sans valence, surajoutés à la construction » de l'infinitif. Elle est proche également de celle de Jacobson (1990), qui, dans une grammaire catégorielle, en fait des foncteurs S/S combinés par composition fonctionnelle avec l'infinitif. Cette représentation permet un parallélisme intéressant entre syntaxe et sémantique, mais laisse entières la question de l'ordre des mots et celle de l'accord avec le sujet.

39. On peut également noter que à et de ne sont analysés ici ni comme tête d'un SP, ni comme complémenteurs (ou marqueurs syntagmatiques). Ce sont plutôt des marqueurs de V, qu'on doit répéter sur chaque verbe en cas de coordination:

(i) Jean commence à lire le grec et à le comprendre

(ii) *Jean commence à lire le grec et le comprendre

40. Les propriétés plus marginales des verbes «à montée » vues supra (2.2.3) s'expliquent aisément. Dans les tours à Inf, c'est l'infinitif qui autorise la construction indépendamment de 
l'adjonction du verbe «à montée ». Pour les constructions «à montée du passif» (achevé d'imprimer), il suffit d'autoriser la forme infinitive du verbe à ancrer l'arbre élémentaire du passif (comme la forme participiale) : le participe passif du verbe "à montée » (s'il existe) s'y adjoint sans complication particulière.

41. Comme cela a été noté par Candito \& Kahane (1998), l'inversion des dépendances sémantiques dans l'arbre de dérivation est un phénomène général en cas d'adjonction (qui s'applique aussi aux modifieurs prédicatifs et aux verbes à complétive). Afin d'identifier les arbres auxiliaires ndant à leur argument (Figure 7).

42. On aurait pu penser à utiliser une différence de catégorie: les auxiliaires de temps s'adjoignant à V et les verbes "à montée " à SV. J'ai expliqué ailleurs pourquoi un nœud SV regroupant verbe et compléments me semblait injustifié en français (Abeillé 1996).

43. Le traitement du en complément de nom nécessite, puisqu'on veut avoir le nom et son complément dans la même structure élémentaire, de considérer cette dernière comme un couple d'arbres, qui doivent se combiner simultanément à deux nœuds différents dans l'arbre du verbe (cf. Abeillé 1998). L'insertion conjointe de en et du sujet dans l'arbre de l'infinitif se fait bien indépendamment de l'adjonction du verbe « à montée ».

44. Une conséquence est que les auxiliaires sont traités en syntaxe, tandis que la flexion verbale est traitée en morphologie.

45. Les deux modèles se sont récemment rapprochés : à l'instar de la multiplication des arbres élémentaires disponibles à l'intérieur d'une famille TAG, HPSG a généralisé l'emploi de règles lexicales (ou de contraintes de correspondance entre descriptions de mots et descriptions de lexèmes) pour toutes les variantes de construction d'un même lexème (et a abandonné les catégories vides); à l'instar de l'organisation hiérarchique des types de mots et des types de syntagmes en HPSG, les grammaires TAG se présentent désormais comme une hiérarchie de descriptions syntagmatiques partielles (Candito 1996, 1999).

46. En HPSG, la tête d'un syntagme n'est pas forcément sa tête sémantique: en cas de modification, le modifieur est la tête sémantique sans être la tête syntaxique.

47. Van Eynde (1994) présente une analyse alternative avec l'auxiliaire comme marqueur et le participe comme tête mais doit modifier les principes habituels de propagation de traits en HPSG. Les conséquences pour l'ensemble du modèle ne sont pas claires.

48. Le modèle est assez souple pour autoriser plusieurs analyses ; si l'on s'en tient aux auxiliaires de temps français, les trois structures imaginables (cf. figure 1 supra) ont été proposées (avec des prédictions différentes) : un complexe verbal (Bratt 1990), un complément SV (Miller 1991), une structure plate (Abeillé \& Godard 1996). Ces trois analyses ont en commun de considérer l'auxiliaire comme la tête.

49. Une analyse avec complexe verbal (et l'auxiliaire comme tête) est plus difficile en français car on voit mal quel statut aurait le $\mathrm{V}$ dominant : s'il est lexical, il ne peut inclure des modifieurs syntagmatiques (qui sont pourtant possibles entre l'auxiliaire et le participe); s'il est syntagmatique, on voit mal comment en exclure les compléments (qui sont eux impossibles entre auxiliaire et participe).

50. La plupart des analyses HPSG maintiennent un SV interne (y compris Abeillé \& Godard 1996) sans justification profonde; je ne le maintiens pas ici pour favoriser la comparaison avec TAG.

51. Les propriétés marginales des verbes « à montée ", c'est-à-dire les tours à Inf ou la " montée » du passif, sont plus difficiles à prendre en compte sauf à supposer une structure plate alternative pour certains verbes "à montée ", qui leur permette d'hériter des compléments (non clitiques) de l'infinitif.

52. Les seuls exceptions au principe sont les explétifs (it, there). Pollard \& Sag n'envisagent pas le cas des arguments idiomatiques non référentiels. On peut penser qu'ils adoptent l'analyse compositionnelle de Nunberg et al. (1994) selon laquelle l'argument figé peut exister en dehors 
de l'expression idiomatique et est sélectionné par des traits particuliers (et donc transmis par unification au verbe " à montée »).

53. Ce principe est critiqué par Baschung (1998) qui propose de le remplacer par un principe de coïndiciation entre argument sans rôle sémantique et sujet non réalisé de l'infinitif. Dans cette proposition, non seulement on perd toute différence syntaxique entre "montée » et "contrôle " (ce qui est voulu par l'auteur) mais on perd aussi toute contrainte de mise en correspondance entre argument syntaxique et argument sémantique.

54. Pollard \& Sag, pour l'anglais, s'appuient sur les données de Jacobson (1990) qui identifie trois déficiences syntaxiques des verbes «à montée » (quiles distinguent des verbes « à contrôle ») :

- pas d'ellipse de l'infinitive (John tried vs * John seems),

- pas de topicalisation de l'infinitive (To go to the beach, John tried vs *To be smart, John seems.),

- pas de pronominalisation de l'infinitive (John tried that. vs *John seems that).

Le fait que certains auxiliaires anglais autorisent les deux premières contructions pose néanmoins problème au principe de montée.

55. Pour les auxiliaires (et les verbes « à montée » non prédicatifs), il faut modifier le principe de montée pour autoriser le cas du participe (ou de l'infinitif) complément sans rôle sémantique. On ajoutera par exemple « ou dans la mesure où cet argument est un complément non saturé dont il hérite le contenu ». L'extraction ou la pronominalisation du complément infinitif d'aller ou venir, qui, comme les auxiliaires être et avoir, héritent du contenu de l'infinitif auquel ils ajoutent une marque de temps, seront donc bien exclues.

56. Miller (1991) propose de donner en cas de conflit la priorité aux critères phonétiques et morphologiques sur les critères sémantiques, c'est ce qui motive son analyse des pronoms faibles et des déterminants comme affixes en français.

\section{RÉSUMÉS}

L'analyse transformationnelle des verbes "à montée», si elle a le mérite de maintenir un principe de correspondance entre arguments syntaxiques et arguments sémantiques, pose plus de problèmes qu'elle n'en résout. L'analyse alternative généralement adoptée en grammaires d'unification, qui identifie les structures syntaxiques des auxiliaires, des verbes à montée et des verbes à contrôle, ne tient pas en français. A partir de ce double constat, cet article propose une troisième voie pour rendre compte des ressemblances et des différences entre verbes «à montée» et auxiliaires, tout en maintenant une coïncidence entre arguments syntaxiques référentiels et arguments sémantiques dans une grammaire d'arbres adjoints.

The (subject-to-subject) raising transformation, while ensuring a mapping principle between syntactic and semantic arguments, raises more questions than it can solve. The alternative analysis proposed by unification-based grammars assigns the same syntactic structure to auxiliaries, raising verbs and control verbs and cannot be true for French. This paper proposes a third solution within Tree Adjoining Grammar which can capture both similarities and differences between auxiliaries and " raising " verbs, while maintaining a coincidence between (referential) syntactic and semantic arguments. 


\section{AUTEUR}

ANNE ABEILLÉ

TALaNa, Université Paris 7 \& IUF 